\title{
Optimal Design of Large Dimensional Adaptive Subspace Detectors
}

\author{
Ismail Ben Atitallah, Abla Kammoun, Mohamed-Slim Alouini, Tareq Y. Al Naffouri
}

\begin{abstract}
This paper addresses the design of Adaptive Subspace Matched Filter (ASMF) detectors in the presence of a mismatch in the steering vector. These detectors are coined as adaptive in reference to the step of utilizing an estimate of the clutter covariance matrix using training data of signalfree observations. To estimate the clutter covariance matrix, we employ regularized covariance estimators that, by construction, force the eigenvalues of the covariance estimates to be greater than a positive scalar $\rho$. While this feature is likely to increase the bias of the covariance estimate, it presents the advantage of improving its conditioning, thus making the regularization suitable for handling high dimensional regimes. In this paper, we consider the setting of the regularization parameter and the threshold for ASMF detectors in both Gaussian and Compound Gaussian clutters. In order to allow for a proper selection of these parameters, it is essential to analyze the false alarm and detection probabilities. For tractability, such a task is carried out under the asymptotic regime in which the number of observations and their dimensions grow simultaneously large, thereby allowing us to leverage existing results from random matrix theory. Simulation results are provided in order to illustrate the relevance of the proposed design strategy and to compare the performances of the proposed ASMF detectors versus Adaptive normalized Matched Filter (ANMF) detectors under mismatch scenarios.
\end{abstract}

Index Terms-Adaptive Normalized Matched Filters, Adaptive Subspace Matched Filters, regularized covariance matrix estimation, robust estimation, detection performance, Random Matrix Theory.

\section{INTRODUCTION}

Detection of targets embedded in noise is a standard problem in radar applications. The detection process is merely based on solving a hypothesis testing problem in order to decide whether a target is likely to be present or not. A large variety of detectors exists depending on their status of knowledge. If the covariance matrix of the noise is unknown, some detectors resort to a two-step Generalized Likelihood Ratio test (GLRT) procedure. This procedure consists of deriving a test statistic when the structure of the covariance matrix is known, then, the unknown covariance matrix is replaced by an estimate that uses pure noise observations. Such detectors are referred to as adaptive detectors. Among them, we distinguish the two-step adaptive matched filter (AMF) [1] and the adaptive normalized matched filter (ANMF) [2], also know as the Adaptive Coherence Estimator (ACE) [3], [4].

This work was funded in part by a CRG2 grant CRG_R2_13_ALOU_KAUST_2 from the Office of Competitive Research (OCRF) at King Abdullah University of Science and Technology (KAUST).

The authors are with the Electrical Engineering Program, King Abdullah University of Science and Technology, Thuwal, Saudi Arabia; e-mail:\{ismail.benatitallah, abla.kammoun, tareq.alnaffouri, slim.alouini\}@kaust.edu.sa.
The aforementioned detectors presume perfect knowledge of the signal steering vector. It has been well-established in the very early works of Kelly [5], [6] that a mismatch in the knowledge of the steering vector causes the ANMF detector to undergo a significant degradation in detection performance, even in the case where the clutter covariance matrix is properly known [7]. Several detectors in the literature have dealt with the imprecise knowledge of the steering vector [8][11]. Among them, we distinguish the subspace matched filter (SMF) detector which assumes that the steering vector lies in a given linear subspace [12]. Further derivations of this detector have been established in [13], [14] but they have been limited so far to the case of Gaussian noise. This is the major reason why in these works the estimation of the noise clutter was based on the sample covariance matrix, known for its suitability for Gaussian patterns. In realistic scenarios, non-Gaussian noise, named also non-Gaussian clutter in radar terminology, is likely to contaminate the useful signal, especially for highresolution radars and when operating with low grazing angles [15]-[19]. These clutters are characterized by heavy-tailed distributions flashing outliers and consequently diminishing the detection performances. A class of estimators, termed robust estimators, used by Huber, Hampel and Maronna [20][22], and extended more recently by Ollila to the complex case [23]-[25], are recognized for their resilience to the drawbacks of heavy tailed-distribution clutters.

Aside from robustness to the presence of outliers, another important question, which should not be underestimated, is the conditioning of the covariance estimate. This feature becomes all the more critical when the number of observations is less than that of the observations' dimension or when the true covariance matrix is ill-conditioned. In order to guarantee an acceptable conditioning, regularized estimators, which originate from the diagonal loading technique of Abramowitch and Calson [26], [27] have been proposed. The idea is simply to force by construction all the eigenvalues of the covariance estimate to be greater than a regularization coefficient $\rho$. We distinguish two commonly used regularized estimators: the regularized sample covariance matrix (RSCM) for Gaussian clutters and the Regularized Tyler estimators (RTE) for nonGaussian clutters. The regularization parameter should be set adequately to fully benefit from the shrinkage technique. This problem has been investigated in the literature, namely in [28], [29] for the RSCM and in [30], [31] for the RTE, by minimizing a certain distance between the regularized estimate and the true covariance matrix. However, these choices might be not optimal in general since they are generic in that they are made irrespective of the considered application. 
In this paper, we consider the setting of the regularization parameter of the RTE and the RSCM when these estimates are used by the adaptive subspace matched filter (ASMF) as replacements of the unknown noise covariance matrix. The corresponding detectors will be coined ASMF-RSCM and ASMF-RTE. Obviously, finding an optimal setting of the regularization coefficient requires a careful analysis of the false alarm and detection probabilities. This seems to be out of reach if exact closed-forms are looked for. Failing that, we will consider the analysis of these quantities under the asymptotic regime in which the number of observation and their dimensions grow simultaneously large. The handling of this regime can be made possible by resorting to advanced tools from random matrix theory and recent advances in asymptotic robust statistics [32]-[35]. After analysing the asymptotic false alarm and detection probabilities, the regularization parameter will be tuned in order to achieve the highest possible detection probability. This approach has recently been applied to the ANMF detector, but only when the steering vector is properly known [36]. It is thus important to assess how errors in the steering vector impact the performances of the design in [36] and whether the proposed ASMF detection schemes achieve a higher robustness to these errors.

The remainder of the paper is organized as follows. In the first section, we introduce the detection problem. Then, we present the two regularized estimators used for teh detection schemes and we review some results about the asymptotic behavior of the RTE. This will pave the way towards the next section where we study the impact of the mismatch on the design of the ANMF detectors of [36]. Hereafter, we propose an optimal design of the ASMF-RSCM and ASMFRTE detectors. Finally, we illustrate using simulations the performances of the proposed ASMF-RSCM and ASMF-RTE designs and compare them with that of the ANMF detectors of [36].

Notations: Throughout this paper, we depict vectors in lowercase boldface letters and matrices in uppercase boldface letters. The notations (. $)^{T}$ and (.)* stand for the transpose and the transpose conjugate respectively while $\operatorname{tr}($.$) and (.)^{-1}$ are the trace and inverse operators. The notation $\|$.$\| stands$ for the Euclidean norm for vectors and for spectral norm for matrices. The statement $X \triangleq Y$ defines the new notation $X$ as being equal to $Y$. The real and the imaginary parts of a complex number are denoted as $\Re($.$) and \Im($.$) . The arrow$ $\stackrel{\text { a.s. }}{\longrightarrow}$ designates almost sure convergence. For a sequence of random vectors $X_{n}, X_{n}=o_{p}(1)$ implies the convergence in probability to zero while $X_{n}=O_{p}(1)$ implies that $X_{n}$ is bounded in probability.

\section{Problem Statement}

We consider the problem of detecting a target signal $\mathbf{p}$ in a complex multivariate measurement $\mathbf{y}$ of size $N \times 1$ corrupted by noise as:

$$
\mathbf{y}=\alpha_{N} \mathbf{p}_{N}+\mathbf{x}
$$

where $\mathbf{x}$ stands for the noise vector and $\alpha_{N}$ is a real scalar related to the SNR.
The signal detection problem turns out to be equivalent to considering the following hypothesis test:

$$
\begin{cases}H_{0} & \mathbf{y}=\mathbf{x} \\ H_{1} & \mathbf{y}=\alpha_{N} \mathbf{p}_{N}+\mathbf{x}\end{cases}
$$

If the clutter $\mathbf{x}$ is Gaussian and its covariance matrix $\mathbf{C}_{N}$ is known up to a constant multiplier, i.e. the structure of the covariance matrix is known but the noise power is unknown, the Generalized Likelihood Ratio (GLRT) for the detection problem results in the following test statistic:

$$
T_{N}=\frac{\left|\mathbf{y}^{*} \mathbf{C}_{N}^{-1} \mathbf{p}\right|^{2}}{\mathbf{p}^{*} \mathbf{C}_{N}^{-1} \mathbf{p y}^{*} \mathbf{C}_{N}^{-1} \mathbf{y}}
$$

The statistic $T_{N}$ represents the squared cosine between the whitened measurement $\mathbf{C}_{N}^{-\frac{1}{2}} \mathbf{y}$ and the whitened signal $\mathbf{C}_{N}^{-\frac{1}{2}} \mathbf{p}$. The popularity of this detector can be easily seen from its many alternative names: the constant false alarm (CFAR) coherent matched subspace detector [14], the normalized matched filter (NMF) [37] and the Linear Quadratic GLRT (LQ-GLRT) [38]. It particularly exhibits good performances in both Gaussian and non-Gaussian clutter [39]. In the sequel, this detector will be referred to as the NMF detector.

The NMF detector yields good performances when the nominal steering vector is perfectly matched to the actual steering vector. However, in realistic scenarios, different types of mismatch are likely to occur:

- Spatial mismatch of the spatial steering vector: the backscattered signal might be slightly deviated from the steered beam. Besides, instruments imprecision, multipath propagation and array calibration uncertainty are all eventual reasons to potentially engender mismatches between the actual steering vector and the nominal steering vector.

- Temporal mismatch of the temporal steering vector: additionally to instruments imprecision, it occurs when the speed of the target is not properly matched to the considered one, thereby yielding Doppler uncertainty.

The NMF detector is sensitive to steering vector mismatches and its detection probability vanishes even for minor mismatches. In order to account for a possible mismatch in the knowledge of $\mathbf{p}$, we assume that in case a signal target is present, the actual received vector is given in reality by:

$$
\mathbf{y}=\alpha_{N} \mathbf{v}_{N}+\mathbf{x}
$$

where $\mathbf{v}_{N}$ is the actual steering vector and is allowed to be different from $\mathbf{p}_{N}$.

In practice, a priori knowledge about how much error can occur to the steering vector is available to the detector. It is thus legitimate to rather assume that the unknown steering vector belong to a known subspace of dimension $r<N$, that is $\mathbf{v}_{N}$ can be written as $\mathbf{v}_{N}=\mathbf{H}_{N} \mathbf{b}$ where $\mathbf{b}$ contains the unknown location of $\mathbf{v}_{N}$ in the subspace spanned by the $r$ columns of $\mathbf{H}_{N}$.

The GLRT of this linear subspace detector yields the following statistic [12]:

$$
S_{N}=\frac{\mathbf{y}^{*} \mathbf{C}_{N}^{-1} \mathbf{H}_{N}\left(\mathbf{H}_{N}^{*} \mathbf{C}_{N}^{-1} \mathbf{H}_{N}\right)^{-1} \mathbf{H}_{N}^{*} \mathbf{C}_{N}^{-1} \mathbf{y}}{\mathbf{y}^{*} \mathbf{C}_{N}^{-1} \mathbf{y}} \gtrless_{H_{0}}^{H_{1}} \gamma
$$


The statistics corresponding to the NMF and SMF detectors suppose that the covariance matrix $\mathbf{C}_{N}$ is known up to a constant multiplier. Since such an assumption does not hold in practice, a natural, though ad hoc approach, consists in replacing in the statistics of the NMF and the SMF the unknown covariance matrix $\mathbf{C}_{N}$ by an estimate built on $n$ training signal free observations $\mathbf{x}_{1}, \cdots, \mathbf{x}_{n}$. The corresponding detectors are thus coined adaptive detectors in reference to the estimation procedure of the covariance matrix.

In this paper, we will study the cases of Gaussian and compound Gaussian clutters. Prior to presenting the methods that will be used for the estimation of the covariance matrix, we shall set out in the following the working assumptions that will be considered for Gaussian and compound Gaussian clutters.

Assumption A-1 (Gaussian clutters). For $i \in\{1, \cdots, n\}$, $\mathbf{x}_{i}=\mathbf{C}_{N}^{\frac{1}{2}} \mathbf{w}_{i}$, with:

- $\mathbf{w}_{1}, \cdots, \mathbf{w}_{n}$ are $N \times 1$ independent standard Gaussian random vectors with zero-mean and covariance $\mathbf{I}_{N}$.

- Matrix $\mathbf{C}_{N} \in \mathbb{C}^{N \times N}$ is such that limsup $\left\|\mathbf{C}_{N}\right\|<\infty$ with $\frac{1}{N} \operatorname{tr} \mathbf{C}_{N}=1$,

Assumption A-2 (Compound Gaussian clutters). For $i \in$ $\{1, \cdots, n\}, \mathbf{x}_{i}=\sqrt{\tau_{i}} \mathbf{C}_{N}^{\frac{1}{2}} \mathbf{w}_{i} \triangleq \sqrt{\tau_{i}} \mathbf{z}_{i}$, with:

- $\mathbf{w}_{1}, \cdots, \mathbf{w}_{n}$ are $N \times 1$ independent standard Gaussian random vectors with zero-mean and covariance $\mathbf{I}_{N}$,

- Matrix $\mathbf{C}_{N} \in \mathbb{C}^{N \times N}$ is such that limsup $\left\|\mathbf{C}_{N}\right\|<\infty$ with $\frac{1}{N} \operatorname{tr} \mathbf{C}_{N}=1$,

- $\tau_{i}>0$ are independent of $\mathbf{w}_{i}$,

It is worth mentioning that the normalization $\frac{1}{N} \operatorname{tr} \mathbf{C}_{N}$ is not limiting since the statistics under consideration are invariant to the scaling of $\mathbf{C}_{N}$. Finally, for technical arguments, we shall consider the following additional assumptions on $\mathbf{p}_{N}$ and $\mathbf{H}_{N}$.

Assumption A-3. Vectors $\left(\mathbf{p}_{N}\right)_{N}$ satisfy the following conditions:

- $\left\|\mathbf{p}_{N}\right\|=1$,

- $\liminf \mathbf{p}_{N}^{*} \mathbf{C}_{N} \mathbf{p}_{N}>0$.

Assumption A-4. Matrices $\left(\mathbf{H}_{N}\right)_{N}$ satisfy the following conditions:

- The columns of $\mathbf{H}_{N}$ are unit norm vectors.

- $\liminf \lambda_{\min }\left(\mathbf{H}_{N}^{*} \mathbf{C}_{N} \mathbf{H}_{N}\right)>0$.

- The rank of $\mathbf{H}_{N}$ is $r$ and does not grow with $N$ and $n$. Moreover, scalars $\left(\alpha_{N}\right)$ satisfy:

$$
0<\liminf \alpha_{N} \leq \lim \sup \alpha_{N}<+\infty .
$$

In the sequel, for sake of simplicity, we shall drop the subscript $N$.

The first two items in Assumption 3 and 4 indicate that a low level of the SNR, defined as the ratio between the gain of the signal of interest and the power of the clutter, is considered. Specifically,

$$
\mathrm{SNR}=\frac{\alpha^{2} \mathbf{p}^{*} \mathbf{p}}{\mathbb{E}\left[\mathbf{x}^{*} \mathbf{x}\right]}=\frac{\alpha^{2}}{N} .
$$

As we will see later, this assumption is essential in order allow inferring useful information about the detection probability.

\section{ESTIMATION OF THE CLUTTER COVARIANCE MATRIX}

As earlier mentioned, the ANMF and ANMF detectors need to acquire a consistent estimate of the noise covariance matrix. In this paper, we consider the use of regularized covariance matrix estimators. Our choice is mainly motivated by the capabilities of this class of estimators to successfully handle the ill-conditioning of the covariance estimate caused by the possible deficiency in observations.

Depending on the nature of the clutter, two regularized covariance matrix estimators will be considered in the sequel, namely the regularized sample covariance matrix (RSCM) and the regularized Tyler estimators (RTE).

\section{A. Regularized Sample Covariance matrix}

In case of Gaussian clutters, we propose to use the regularized sample covariance matrix defined as:

$$
\widehat{\mathbf{R}}_{N}(\rho)=(1-\rho) \widehat{\mathbf{R}}_{N}+\rho \mathbf{I}_{N}
$$

where $\widehat{\mathbf{R}}_{N}$ corresponds to the traditional sample covariance matrix (SCM) estimator given by:

$$
\widehat{\mathbf{R}}_{N}=\frac{1}{n} \sum_{i=1}^{n} \mathbf{x}_{i} \mathbf{x}_{i}^{*} .
$$

The regularization parameter $\rho \in[0,1]$ serves to give more or less credence to the sample covariance matrix $\widehat{\mathbf{R}}_{N}$ depending on the available number of samples. The rationale behind this choice essentially lies in that, for Gaussian clutters, the SCM does correspond to the maximum likelihood estimator of the covariance matrix, hence the prospect for the RSCM to achieve good performances while exhibiting improved conditioning.

As per the ad hoc design procedure of radar detectors, the RSCM obtained from (1) will serve as a replacement of the unknown covariance matrix $\mathbf{C}_{N}$ in $T_{N}$ and $S_{N}$, thereby yielding the following statistics $\widehat{T}_{N}^{\mathrm{RSCM}}$ and $\widehat{S}_{N}^{\mathrm{RSCM}}$ :

$$
\begin{aligned}
& \widehat{T}_{N}^{\mathrm{RSCM}}(\rho)=\frac{\left|\mathbf{y}^{*} \widehat{\mathbf{R}}_{N}^{-1}(\rho) \mathbf{p}\right|^{2}}{\mathbf{p}^{*} \widehat{\mathbf{R}}_{N}^{-1}(\rho) \mathbf{p y} \mathbf{y}^{*} \widehat{\mathbf{R}}_{N}^{-1}(\rho) \mathbf{y}}, \\
& \widehat{S}_{N}^{\mathrm{RSCM}}=\frac{\mathbf{y}^{*} \widehat{\mathbf{R}}_{N}^{-1}(\rho) \mathbf{H}\left(\mathbf{H}^{*} \widehat{\mathbf{R}}_{N}^{-1}(\rho) \mathbf{H}\right)^{-1} \mathbf{H}^{*} \widehat{\mathbf{R}}_{N}^{-1}(\rho) \mathbf{y}}{\mathbf{y}^{*} \widehat{\mathbf{R}}_{N}^{-1}(\rho) \mathbf{y}}
\end{aligned}
$$

The corresponding detectors will be referred to as ANMFRSCM and ASMF-RSCM.

\section{B. Regularized Tyler estimator}

The use of the RSCM for non-Gaussian heavy tailed clutters would lead to poor performances because, as a derivative of the SCM, it inherits its main major limitation of being vulnerable to the presence of outliers. In order to estimate the covariance matrix of heavy tailed clutters, it is sensible to resort instead to the class of robust-scatter estimators, known for their high resilience to impulsive noise. The quest for improving the conditioning of covariance matrix estimates by using the artifice of regularization has recently led to the emergence of regularized versions of robust estimators. Of 
interest in the present work is the regularized Tyler estimator (RTE), which was originally proposed in [40].

For $\rho \in\left(\max \left\{0,1-\frac{n}{N}\right\}, 1\right]$, the RTE is defined as the unique solution to the following equation:

$$
\widehat{\mathbf{C}}_{N}(\rho)=(1-\rho) \frac{1}{n} \sum_{i=1}^{n} \frac{\mathbf{x}_{i} \mathbf{x}_{i}^{*}}{\frac{1}{N} \mathbf{x}_{i}^{*} \widehat{\mathbf{C}}_{N}^{-1}(\rho) \mathbf{x}_{i}}+\rho \mathbf{I}_{N}
$$

Note that the definition of the RTE is reminiscent of the robust Tyler estimator of scatter [41] and the Ledoit-Wof's shrinkage estimator [28]. In contrast to the wide majority of existing robust estimators, it is well-defined when $N$ is less than the number of observations $n$ as long as $\rho \geq 1-\frac{n}{N}$. It is also worth mentioning that by changing the regularization parameter $\rho$, one can smoothly move from the unbiased Tyler estimator [42] $(\rho=0)$ to the identity matrix $(\rho=1)$, which represents a crude estimate of the unknown covariance matrix $\mathbf{C}_{N}$.

With the RTE at hand, we define similarly to the previous section the following decision statistics, which are obtained by replacing in $T_{N}$ and $S_{N}$ the unknown covariance matrix by $\widehat{\mathbf{C}}_{N}(\rho)$ :

$$
\begin{aligned}
& \widehat{T}_{N}^{\mathrm{RTE}}(\rho)=\frac{\left|\mathbf{y}^{*} \widehat{\mathbf{C}}_{N}^{-1}(\rho) \mathbf{p}\right|^{2}}{\mathbf{p}^{*} \widehat{\mathbf{C}}_{N}^{-1}(\rho) \mathbf{p y}{ }^{*} \widehat{\mathbf{C}}_{N}^{-1}(\rho) \mathbf{y}}, \\
& \widehat{S}_{N}^{\mathrm{RTE}}=\frac{\mathbf{y}^{*} \widehat{\mathbf{C}}_{N}^{-1}(\rho) \mathbf{H}\left(\mathbf{H}^{*} \widehat{\mathbf{C}}_{N}^{-1}(\rho) \mathbf{H}\right)^{-1} \mathbf{H}^{*} \widehat{\mathbf{C}}_{N}^{-1}(\rho) \mathbf{y}}{\mathbf{y}^{*} \widehat{\mathbf{C}}_{N}^{-1}(\rho) \mathbf{y}} .
\end{aligned}
$$

The corresponding detectors will be thus termed ANMF-RTE and ASMF-RTE.

\section{Setting of the regularization parameter}

While the regularization parameter $\rho$ is expected to enhance the conditioning of the used covariance matrix estimates, a special care must be paid when it comes to selecting its value. Obviously, a high value of $\rho$ tends to produce estimates with higher conditioning but it might at the same time cause the covariance estimate to deviate from the true unknown covariance matrix $\mathbf{C}_{N}$. Most of the previous recent works dealing with the question of setting the regularization parameter usually propose to select for it the value that minimizes a certain distance function between the covariance estimate and the true covariance matrix $\mathbf{C}_{N}$ [29], [30], [43]. While this approach sounds compliant with common sense, it presents the drawback of being performed regardless of the application under consideration. A more relevant choice that is appropriate for radar detection applications would be to select the values of the regularization parameter that maximize the detection probability while keeping constant the false alarm probability. The major difficulty towards applying this approach is that it necessitates a careful analysis of the distribution of the decision statistics. Under the assumption of fixed values for $N$ and $n$, such a task seems to be out of reach even for the Gaussian clutter case. The intricacy of handling the impact of the covariance matrix estimate on the distribution of the statistic decision has recently led the authors of [36] to consider the asymptotic regime wherein the number of observations $n$ and their dimensions $N$ grow to infinity with $c_{N} \triangleq \frac{N}{n}$ tending to $c$. The motivation behind considering this regime is essentially driven by the existing load of results dealing with the asymptotic behaviour of $\widehat{\mathbf{R}}_{N}(\rho)$ and more recently that of $\widehat{\mathbf{C}}_{N}(\rho)$ [33]. These results have allowed the authors to derive the asymptotic false alarm and detection probabilities for the ANMF detector when the steering vector $\mathbf{p}$ is correctly known (No mismatch). Based on the obtained results, they proposed to set the regularization parameter $\rho$ to the value that maximizes the asymptotic detection probability for a constant level of the asymptotic false alarm probability. Despite being based on asymptotic results, this setting of $\rho$ was found to outperform many of the existing conventional settings of the regularization parameter, even when $N$ and $n$ are not so large. Encouraged by the good performances of this procedure, we extend the work of [36] to handle the possibility of a mismatch between the actual steering vector $\mathbf{v}$ and its value $\mathbf{p}$ assumed by the detector. In particular, we study the impact of the mismatch on the performance of the detectors of [36]. We show that an inaccurate knowledge of the actual steering vector can lead to lower performances, thereby calling for the need to shift the focus towards the use of ASMF based detectors. Similar to the case of ANMF detectors, the good performances of ASMF based detection schemes require the designer to select a proper choice for the regularization parameter. Such a task represents the main contribution of our work and will be performed by building on the approach of [36]. In order to prepare the ground for the exposition of our findings, we will need to review some of the results on the asymptotic behaviour of the RTE. This will constitute the objective of the next section.

\section{Asymptotic behaviour of the RTE}

Recall that the RTE is defined, for $\rho \in\left(\max \left\{0,1-\frac{n}{N}\right\}, 1\right]$, as the unique solution to the following equation:

$$
\widehat{\mathbf{C}}_{N}(\rho)=(1-\rho) \frac{1}{n} \sum_{i=1}^{n} \frac{\mathbf{x}_{i} \mathbf{x}_{i}^{*}}{\frac{1}{N} \mathbf{x}_{i}^{*} \widehat{\mathbf{C}}_{N}^{-1}(\rho) \mathbf{x}_{i}}+\rho \mathbf{I}_{N} .
$$

This equation clearly unveils the difficulty that poses the analysis of the RTE. It shows that in contrast to the RSCM, the RTE does not possess a closed-form expression. Moreover, the dependence between the outer-products in its expression is non-linear, thereby precluding the use of standard tools of random matrix theory. Based on a different rewriting of the robust-scatter estimators, Couillet et al. have recently showed in [33] that $\widehat{\mathbf{C}}_{N}(\rho)$ can be replaced at first order by an equivalent random matrix that follows a classical model for which many results from random matrix theory can be applied. More specifically, consider $\gamma_{N}(\rho)$ the unique solution to:

$$
1=\int \frac{t}{\gamma_{N}(\rho) \rho+(1-\rho) t} \nu_{N}(d t)
$$

and define $\widehat{\mathbf{S}}_{N}(\rho)$ as:

$$
\widehat{\mathbf{S}}_{N}(\rho)=\frac{1}{\gamma_{N}(\rho)} \frac{1-\rho}{1-(1-\rho) c_{N}} \frac{1}{n} \sum_{i=1}^{n} \mathbf{z}_{i} \mathbf{z}_{i}^{*}+\rho \mathbf{I}_{N}
$$

where $\mathbf{z}_{i}$ 's are as defined in Assumption 2 Then, $\widehat{\mathbf{C}}_{N}(\rho)$ is equivalent to $\widehat{\mathbf{S}}_{N}(\rho)$ in the sense of the following Theorem: 
Theorem 1 ( [33]). For any $\kappa>0$ small, define $\mathcal{R}_{\kappa}^{\mathrm{RTE}} \triangleq$ $\left[\kappa+\max \left(0,1-c^{-1}\right), 1\right]$. Then, as $N, n \rightarrow+\infty$ with $c_{N} \rightarrow$ $c \in(0, \infty)$, we have:

$$
\sup _{\rho \in \mathcal{R}_{\kappa}^{\mathrm{RTE}}}\left\|\widehat{\mathbf{C}}_{N}(\rho)-\widehat{\mathbf{S}}_{N}(\rho)\right\| \stackrel{\text { a.s. }}{\longrightarrow} 0 .
$$

The convergence in the operator norm of the difference $\widehat{\mathbf{C}}_{N}(\rho)-\widehat{\mathbf{S}}_{N}(\rho)$ that is shown in Theorem 1 can be of major interest in practice, allowing to carry the analysis of the first order behavior of functionals of $\widehat{\mathbf{S}}_{N}(\rho)$ to that of $\widehat{\mathbf{C}}_{N}(\rho)$. Even though this result can be easily refined as:

$$
\sup _{\rho \in \mathcal{R}_{\kappa}^{\mathrm{RTE}}} N^{\frac{1}{2}-\epsilon}\left\|\widehat{\mathbf{C}}_{N}(\rho)-\widehat{\mathbf{S}}_{N}(\rho)\right\| \stackrel{\text { a.s. }}{\longrightarrow} 0
$$

for each $\epsilon>0$, it does not suffice to study the fluctuations of the commonly used functionals of the covariance matrix, namely the bilinear forms arising in our work. The reason essentially lies in the fluctuations of bilinear forms being at order of $N^{-\frac{1}{2}}$. A further refinement of (3) is, in our opinion, not possible. Fortunately, the inherent structure of bilinear forms leads to faster fluctuations as it is shown in the following proposition:

Proposition 2 ( [35]). Let $\mathbf{a}, \mathbf{b} \in \mathbb{C}^{N}$ with $\|\mathbf{a}\|=\|\mathbf{b}\|=1$ deterministic or random vectors independent of $\mathbf{x}_{1}, \cdots, \mathbf{x}_{n}$. Then, under Assumption 2 as $N, n \rightarrow+\infty$, with $c_{N} \rightarrow c \in$ $(0, \infty)$, for any $\epsilon>0$ and every $k \in \mathbb{Z}$,

$$
\sup _{\rho \in \mathcal{R}_{\kappa}^{\mathrm{RTE}}} N^{1-\epsilon}\left|\mathbf{a}^{*} \widehat{\mathbf{C}}_{N}^{k}(\rho) \mathbf{b}-\mathbf{a}^{*} \widehat{\mathbf{S}}_{N}^{k}(\rho) \mathbf{b}\right| \stackrel{\text { a.s. }}{\longrightarrow} 0 .
$$

The result of Proposition 2 achieves a first step in handling bilinear forms involving the RTE estimator by transferring the study of the fluctuations of the seemingly intractable bilinear form $\mathbf{a}^{*} \widehat{\mathbf{C}}_{N}^{k}(\rho) \mathbf{b}$ to that of $\mathbf{a}^{*} \widehat{\mathbf{S}}_{N}^{k}(\rho) \mathbf{b}$. This will essentially make it of valuable help with the analysis of the fluctuations of the statistics $\widehat{T}_{N}^{\mathrm{RTE}}$ and $\widehat{S}_{N}^{\mathrm{RTE}}$.

\section{Performance of RADAR DETECTORS UNDER MISMATCH}

The aim of this section is to study the performance detection of the ANMF and the ASMF based detectors when the steering vector is not properly known. It is worth mentioning that, for the ANMF detector, while our analysis extends the work in [36 by taking into account the possible presence of a mismatch in the knowledge of the steering vector, it follows straighforwardly from the same calculations of [36] by replacing only one occurrence of the actual vector $\mathbf{p}$ by vector $\mathbf{v}$. These results will be thus provided for sake of clarity without any proofs. The performance analysis of the ASMF detector is, however, much more involved. It constitutes thus the major contribution of the present work.

Prior to exposing the performance analysis for the ANMF and ASMF based detectors, we shall introduce some key deterministic quantities that will arise naturally when using results from random matrix theory.
Denote for $z \in \mathbb{C} \backslash \mathbb{R}_{+}$by $m_{N}(z)$ the unique complex solution to:

$$
\begin{aligned}
m_{N}(z) & =\left(-z+c_{N}(1-\rho)\right. \\
& \left.\times \frac{1}{N} \operatorname{tr} \mathbf{C}_{N}\left(\mathbf{I}_{N}+(1-\rho) m_{N}(z) \mathbf{C}_{N}\right)^{-1}\right)^{-1}
\end{aligned}
$$

that satisfies $\Im(z) \Im\left(m_{N}(z)\right) \geq 0$. The existence and uniqueness of $m_{N}(z)$ is standard result from random matrix theory [44]. In our case, it helps characterize the asymptotic behaviour of the empirical measure of the random matrix $(1-\rho) \frac{1}{n} \sum_{i=1}^{n} \mathbf{x}_{i} \mathbf{x}_{i}^{*}$ [36]. 11 Define also matrix $\mathbf{Q}(z)$ given by $\mathbf{Q}(z) \triangleq\left(\mathbf{I}_{N}+(1-\rho) m_{N}(-z) \mathbf{C}_{N}\right)^{-1}$. This matrix will essentially serve to approximate traces and quadratic forms involving the random matrix $\widehat{\mathbf{R}}_{N}^{-1}(\rho)$. In particular, we have for any deterministic matrix $\mathbf{U}_{N}$ with uniformly bounded spectral norm:

$$
\frac{1}{N} \operatorname{tr} \mathbf{U} \widehat{\mathbf{R}}_{N}^{-1}(\rho)-\frac{1}{\rho N} \operatorname{tr} \mathbf{U} \mathbf{Q}_{N}(\rho) \stackrel{\text { a.s. }}{\longrightarrow} 0
$$

and any unit norm vectors $\mathbf{a}$ and $\mathbf{b}$ :

$$
\mathbf{a}^{*} \widehat{\mathbf{R}}_{N}^{-1}(\rho) \mathbf{b}-\frac{1}{\rho} \mathbf{a}^{*} \mathbf{Q}_{N}(\rho) \mathbf{b} \stackrel{\text { a.s. }}{\longrightarrow} 0 .
$$

Furthermore, as we will see later, the asymptotic results that will be derived for the Gaussian case can be transferred using Proposition 2 to that of compound Gaussian clutters, by performing a change of variable in $\rho$ :

$$
\rho \leftrightarrow \underline{\rho} \triangleq \rho\left(\rho+\frac{1}{\gamma_{N}(\rho)} \frac{1-\rho}{1-(1-\rho) c}\right)^{-1} .
$$

Finally, define for $\kappa>0, \mathcal{R}_{\kappa}^{\mathrm{RSCM}}$ and $\mathcal{R}_{\kappa}^{\mathrm{RTE}}$, the intervals swept by the regularization parameter $\rho$ when a RSCM or a RTE is used, as:

$$
\begin{aligned}
\mathcal{R}_{\kappa}^{\mathrm{RSCM}} & =[\kappa, 1] \\
\mathcal{R}_{\kappa}^{\mathrm{RTE}} & =\left[\kappa+\max \left(0,1-c^{-1}\right), 1\right] .
\end{aligned}
$$

With these notations at hand, we are now in position to handle the analysis of ANMF based detectors. As earlier mentioned, results for the ANMF detector will be provided without proofs because they directly follow along the same lines of [36].

\section{A. ANMF detectors with mismatched steering vector}

This section extends the results of [36] about the false alarm and detection probabilities of the ANMF detectors by allowing for a possible mismatch in the steering vector. It also illustrates the role of these results in allowing for an appropriate design procedure of the detection schemes.

We recall that the ANMF detector is coined ANMF-RSCM if used over Gaussian clutters and ANMF-RTE if operating over compound Gaussian clutters. The derivation of the

\footnotetext{
${ }^{1}$ Let $\hat{\nu}_{N}=\frac{1}{N} \sum_{i=1}^{N} \delta_{\lambda_{i}}$ be the empirical measure of the random matrix $\frac{1-\rho}{n} \sum_{i=1}^{n} \mathbf{x}_{i} \mathbf{x}_{i}^{*}$ with $\lambda_{1}, \cdots, \lambda_{N}$ being the eigenvalues of $\frac{1-\rho}{n} \sum_{i=1}^{n} \mathbf{x}_{i} \mathbf{x}_{i}^{*}$. Denote by $\hat{m}_{N}(z)$ its Stieltjes transform given by $\hat{m}_{N}(z)=\int(t-z)^{-1} \hat{\nu}_{N}(d t)=\frac{1}{N} \sum_{i=1}^{N} \frac{1}{\lambda_{i}-z}$. Then, quantity $m_{N}(z)$ is the Stieljes transform of a certain deterministic measure $\mu_{N}$, (i.e, $m_{N}(z)=$ $\int(t-z)^{-1} \mu_{N}(d t)$ ) which approximates in the almost sure sense $\hat{m}_{N}(z)$ (i.e $\hat{m}_{N}(z)-m_{N}(z) \stackrel{\text { a.s. }}{\longrightarrow} 0$.).
} 
false alarm and detection probabilities for the ANMF-RSCM and ANMF-RTE is carried out under the asymptotic regime wherein $N, n \rightarrow+\infty$ with $c_{N}=\frac{N}{n} \rightarrow c$. In particular, the following theorem provides in closed-form the expression of the asymptotic false alarm probability.

Theorem 3 (False alarm probability). 1) Gaussian clutters: Assume that the primary data $\mathbf{y}$ and secondary data $\mathbf{x}_{1}, \cdots, \mathbf{x}_{n}$ are distributed as the $\mathbf{x}_{i}$ 's in Assumption 1. Then, as $N, n \rightarrow \infty$ with $c_{N} \rightarrow c \in(0, \infty)$

$$
\begin{aligned}
\sup _{\rho \in \mathcal{R}_{\kappa}^{\mathrm{RSCM}}} \mid \mathbb{P}\left[\widehat{T}_{N}^{\mathrm{RSCM}}(\rho)>\frac{t}{N} \mid H_{0}\right] \\
-\exp \left(-\frac{t}{2 \sigma_{N, \mathrm{SCM}}^{2}(\rho)}\right) \mid \stackrel{\text { a.s. }}{\longrightarrow} 0
\end{aligned}
$$

where:

$$
\begin{aligned}
\sigma_{N, \mathrm{SCM}}^{2}(\rho) & \triangleq \frac{1}{2} \frac{\mathbf{p}^{*} \mathbf{C}_{N} \mathbf{Q}_{N}^{2}(\rho) \mathbf{p}}{\mathbf{p}^{*} \mathbf{Q}_{N}(\rho) \mathbf{p} \frac{1}{N} \operatorname{tr} \mathbf{C}_{N} \mathbf{Q}_{N}(\rho)} \\
& \times \frac{1}{1-c(1-\rho)^{2} m_{N}(-\rho)^{2} \frac{1}{N} \operatorname{tr} \mathbf{C}_{N}^{2} \mathbf{Q}_{N}^{2}(\rho)}
\end{aligned}
$$

2) Compound Gaussian clutters: Assume that the primary data $\mathbf{x}$ and secondary data $\mathbf{x}_{1}, \cdots, \mathbf{x}_{n}$ are distributed as the $\mathbf{x}_{i}$ 's in Assumption 2 Then, as $N, n \rightarrow \infty$ with $c_{N} \rightarrow c \in(0, \infty)$

$$
\begin{aligned}
\sup _{\rho \in \mathcal{R}_{\kappa}^{\mathrm{RSCM}}} \mid \mathbb{P}\left[\widehat{T}_{N}^{\mathrm{RTE}}(\rho)>\frac{t}{N} \mid H_{0}\right] \\
-\exp \left(-\frac{t}{2 \sigma_{N, \mathrm{RTE}}^{2}(\rho)}\right) \mid \stackrel{\text { a.s. }}{\longrightarrow} 0
\end{aligned}
$$

where:

$$
\begin{aligned}
& \sigma_{N, \mathrm{RTE}}^{2}(\rho) \triangleq \frac{1}{2} \frac{\mathbf{p}^{*} \mathbf{C}_{N} \mathbf{Q}_{N}^{2}(\underline{\rho}) \mathbf{p}}{\mathbf{p}^{*} \mathbf{Q}_{N}(\underline{\rho}) \mathbf{p} \frac{1}{N} \operatorname{tr} \mathbf{C}_{N} \mathbf{Q}_{N}(\underline{\rho})} \\
& \times \frac{1}{1-c(1-\underline{\rho})^{2} m_{N}(-\underline{\rho})^{2} \frac{1}{N} \operatorname{tr} \mathbf{C}_{N}^{2} \mathbf{Q}_{N}^{2}(\underline{\rho})} \\
& \text { and } \underline{\rho}=\rho\left(\rho+\frac{1}{\gamma_{N}(\rho)} \frac{1-\rho}{1-(1-\rho) c}\right)
\end{aligned}
$$

Theorem 3 establishes that the statistic $\widehat{T}_{N}^{\mathrm{RSCM}}(\rho)$ and $\widehat{T}_{N}^{\mathrm{RTE}}(\rho)$ behave as exponentially distributed random variables with parameter $\frac{1}{2 \sigma_{N, \mathrm{SCM}^{2}(\rho)}}$ and $\frac{1}{2 \sigma_{N, \mathrm{RTE}^{2}(\rho)}}$. Note that the expression of the asymptotic false alarm probability is independent of the actual vector $\mathbf{v}$ because the received signal vectors used in the statistics $\widehat{T}_{N}^{\mathrm{RSCM}}$ and $\widehat{T}_{N}^{\mathrm{RTE}}$ correspond to purely noise signals. The impact of the mismatch in the knowledge of the steering vector will, however, arise in the expression of the probability of detection as shown by the following Theorem.

Theorem 4 (Detection probability). 1) Gaussian clutters: Assume that the primary data $\mathbf{y}=\alpha \mathbf{p}+\mathbf{x}$, where $\alpha$ and $\mathbf{p}$ satisfy Assumption 3 and $\mathbf{x}$ is distributed as the $\mathbf{x}_{i}$ 's in Assumption 1 . Assume also that $\|\mathbf{v}\|=1$. Then, as
$N, n \rightarrow \infty$ with $c_{N} \rightarrow c$, we have for any $\kappa>0$,

$$
\begin{aligned}
& \sup _{\rho \in \mathcal{R}_{\kappa}^{\mathrm{SCM}}} \mid \mathbb{P}\left[\widehat{T}_{N}^{\mathrm{RSCM}}(\rho)>\frac{t}{N} \mid H_{1}\right] \\
& -Q_{1}\left(g_{\mathrm{SCM}}(\mathbf{p}, \mathbf{v}), \frac{\sqrt{t}}{\sigma_{N, \mathrm{SCM}}(\rho)}\right) \mid \stackrel{\text { a.s. }}{\longrightarrow} 0 .
\end{aligned}
$$

where $Q_{1}$ is the Marcum $Q$-function ${ }^{2}$ while $\sigma_{N, \mathrm{SCM}}$ has the same expression as in Theorem 3 and $g(\mathbf{p}, \mathbf{v})$ is given by:

$$
\begin{aligned}
g_{S C M}(\mathbf{p}, \mathbf{v}) & =\frac{\sqrt{1-c(1-\rho)^{2} m(-\rho)^{2} \frac{1}{N} \operatorname{tr} \mathbf{C}_{N}^{2} \mathbf{Q}_{N}^{2}(\rho)}}{\sqrt{\mathbf{p}^{*} \mathbf{C}_{N} \mathbf{Q}_{N}^{2}(\rho) \mathbf{p}}} \\
& \times \sqrt{2} a\left|\mathbf{p}^{*} \mathbf{Q}_{N}(\rho) \mathbf{v}\right| .
\end{aligned}
$$

2) Compound Gaussian clutters: Assume that the primary data $\mathbf{y}=\alpha \mathbf{p}+\mathbf{x}$, where $\alpha$ and $\mathbf{p}$ satisfy Assumption 3 and $\mathbf{x}$ is distributed as the $\mathbf{x}_{i}$ 's in Assumption 2 Assume also that $\|\mathbf{v}\|=1$. Then, as $N, n \rightarrow \infty$ with $c_{N} \rightarrow c$, we have for any $\kappa>0$,

$$
\begin{aligned}
& \sup _{\rho \in \mathcal{R}_{\kappa}^{\mathrm{RTE}}} \mid \mathbb{P}\left[\widehat{T}_{N}^{\mathrm{RTE}}(\rho)>\frac{t}{\sqrt{N}} \mid H_{1}\right] \\
& -\mathbb{E}\left[Q_{1}\left(g_{\mathrm{RTE}}(\mathbf{p}), \frac{\sqrt{t}}{\sigma_{N, \operatorname{RTE}}(\rho)}\right)\right] \mid \rightarrow 0,
\end{aligned}
$$

where the expectation is taken over the distribution of $\tau$, $\sigma_{N, \operatorname{RTE}}(\rho)$ has the same expression as in Theorem 3 and

$$
\begin{aligned}
g_{\mathrm{RTE}}(\mathbf{p}) & =\frac{\sqrt{1-c(1-\underline{\rho})^{2} m(-\underline{\rho}) \frac{1}{N} \operatorname{tr} \mathbf{C}_{N}^{2} \mathbf{Q}_{N}^{2}(\underline{\rho})}}{\sqrt{\mathbf{p}^{*} \mathbf{C}_{N} \mathbf{Q}_{N}^{2}(\underline{\rho}) \mathbf{p}}} \\
& \times \sqrt{\frac{2}{\tau} a\left|\mathbf{p}^{*} \mathbf{Q}_{N}(\underline{\rho}) \mathbf{v}\right| .}
\end{aligned}
$$

and $Q_{1}$ is the Marcum $Q$-function.

Theorem 4 provides the expression of the asymptotic probability of detection when the actual steering vector is different from that assumed by the detector. It is worth mentioning that we obtain the same expression as the one corresponding to the non-mismatched case [36], with the slight difference of $\mathbf{p}^{*} \mathbf{Q}_{N}(\rho) \mathbf{p}$ and $\mathbf{p}^{*} \mathbf{Q}_{N}(\underline{\rho}) \mathbf{p}$ being replaced by $\left|\mathbf{p}^{*} \mathbf{Q}_{N}(\rho) \mathbf{v}\right|$ and $\left|\mathbf{p}^{*} \mathbf{Q}_{N}(\underline{\rho}) \mathbf{v}\right|$. Since $\mathbf{p}^{*} \mathbf{Q}_{N}(\rho) \mathbf{v} \leq \mathbf{p}^{*} \mathbf{Q}_{N}(\rho) \mathbf{p}$ and $\mathbf{p}^{*} \mathbf{Q}_{N}(\rho) \mathbf{v} \leq \mathbf{p}^{*} \mathbf{Q}_{N}(\rho) \mathbf{p}$, a reduction in the first argument of the marcum-Q function occurs, thereby inducing a loss in the probability of detection.

We will now discuss the choice of the regularization parameter and the threshold $t$. As per the spirit of radar detection theory, these values should be set in such a way to keep the asymptotic false alarm probability constant at a given level $\eta$ while maximizing the asymptotic probability of detection. Theorem 3 indicates that such values should satisfy:

$$
\frac{\sqrt{t}}{\sigma_{N, \mathrm{SCM}}(\rho)}=\sqrt{-2 \log \eta}
$$

\footnotetext{
${ }^{2}$ The Marcum-Q function is given by $Q_{1}(a, b)=$ $\int_{b}^{+\infty} x \exp \left(-\frac{x^{2}+a^{2}}{2}\right) I_{0}(a x) d x$ where $I_{0}$ is the 0 order modified
} bessel function of the first kind. 
for the ANMF-RSCM and

$$
\frac{\sqrt{t}}{\sigma_{N, \operatorname{RTE}}(\rho)}=\sqrt{-2 \log \eta}
$$

for the ANMF-RTE. Out of these choices, we need to pick up those values that maximize the asymptotic probability of detection that is expected by the detector. Since the second argument of $Q_{1}$ should be kept fixed in order to approximately guarantee the required level of false alarm probability, the optimization of the detection probability boils down to considering the values for $\rho$ that maximize the second argument. This is equivalent to considering the values in the set:

$$
\rho \in \operatorname{argmax}\left\{f_{\mathrm{SCM}}(\rho)\right\}
$$

for the ANMF-RSCM, and those in the set:

$$
\rho \in \operatorname{argmax}\left\{f_{\mathrm{RTE}}(\rho)\right\}
$$

for the ANMF-RTE, where:

$$
\begin{aligned}
& f_{\mathrm{SCM}}(\rho) \triangleq \frac{1}{2 a^{2}} g_{\mathrm{SCM}}^{2}(\mathbf{p}, \mathbf{p}) \\
& =\frac{\left(1-c(1-\rho)^{2} m(-\rho)^{2} \frac{1}{N} \operatorname{tr} \mathbf{C}_{N}^{2} \mathbf{Q}_{N}^{2}(\rho)\right)\left(\mathbf{p}^{*} \mathbf{Q}_{N}(\rho) \mathbf{p}\right)^{2}}{\mathbf{p}^{*} \mathbf{C}_{N} \mathbf{Q}_{N}^{2}(\rho) \mathbf{p}}
\end{aligned}
$$

and

$$
\begin{aligned}
& f_{\mathrm{RTE}}(\rho) \triangleq \frac{\tau}{2 a^{2}} g_{\mathrm{RTE}}^{2}(\mathbf{p}, \mathbf{p}) \\
& =\frac{\left(1-c(1-\underline{\rho})^{2} m(-\underline{\rho})^{2} \frac{1}{N} \operatorname{tr} \mathbf{C}_{N}^{2} \mathbf{Q}_{N}^{2}(\underline{\rho})\right)\left(\mathbf{p}^{*} \mathbf{Q}_{N}(\underline{\rho}) \mathbf{p}\right)^{2}}{\mathbf{p}^{*} \mathbf{C}_{N} \mathbf{Q}_{N}^{2}(\underline{\rho}) \mathbf{p}}
\end{aligned}
$$

The design procedure described above for the selection of $\rho$ and $t$ is not applicable in practice, since it requires the detector to be fed with the true covariance matrix $\mathbf{C}_{N}$ in order to compute the expressions of $f_{S C M}$ and $\sigma_{N, S C M}$ for the ANMF-RSCM and $f_{R T E}$ and $\sigma_{N, R T E}$ for the ANMFRTE. To overcome this difficulty, consistent estimates for these quantities were provided in [36]. Using these estimates, one can tune the values of the regularization parameter and the threshold in order to meet the requirement of the false alarm probability while maximizing the detection probability. Considering the ANMF-RSCM for example and letting $\hat{f}_{S C M}$ and $\hat{\sigma}_{N, S C M}$ be consistent estimates for $f_{S C M}$ and $\sigma_{N, S C M}$, the design procedure is given by:

- First, set the regularization parameter to the one of the values maximizing $\hat{f}_{\mathrm{SCM}}(\rho)$ :

$$
\hat{\rho}_{N}^{*} \in \arg \max _{\rho \in \mathcal{R}_{\kappa}}\left\{\hat{f}_{\mathrm{SCM}}(\rho)\right\}
$$

- Second, select the threshold $\hat{t}$ as:

$$
\hat{t}=\sigma_{N, \mathrm{SCM}}\left(\hat{\rho}_{N}^{*}\right) \sqrt{-2 \log \eta}
$$

\section{B. ASMF detectors with mismatched steering vector}

As we have previously seen, a mismatch in the array steering vector causes a degradation in the detection performances of the ANMF detectors. This lies behind our major motivation towards investigating the design of ASMF based detectors, known for their high resilience to deviations from the true steering vector. Similar to the ANMF detectors, the design procedure essentially involves the proper selection of the regularization parameter $\rho$ and the threshold value $t$ in order to achieve high detection probability while keeping the false alarm probability at a desired value. To this end, we need to derive in the same way as in the case of ANMF detectors, the expressions for the asymptotic false alarm and detection probabilities.

The following Theorem presents our main results providing the asymptotic false alarm and detection probabilities for both Gaussian and compound-Gaussian clutters. In order to unify the exposition of our results, we will use the notation $H_{\beta}$, with $\beta$ taking the values 0 or 1 , to refer to $H_{0}(\beta=0)$ or $H_{1}(\beta=1)$.

Theorem 5 (False alarm and detection probabilities). 1) Gaussian clutters: As $N, n \rightarrow \infty$ with $c_{N} \rightarrow c \in(0, \infty)$ and $\beta \in\{0,1\}$,

$$
\begin{aligned}
& \sup _{\rho \in \mathcal{R}_{\kappa}^{R S C M}} \mid \mathbb{P}\left[\widehat{S}_{N}^{\mathrm{RSCM}}(\rho)>\frac{t}{N} \mid H_{\beta}\right] \\
& -\mathbb{P}\left[\left(\mathbf{u}^{*} \boldsymbol{\Sigma}(\rho)^{\frac{1}{2}}+\beta \alpha \boldsymbol{\mu}(\rho)^{*}\right) \mathbf{A}(\rho)\right. \\
& \left.\times\left(\boldsymbol{\Sigma}(\rho)^{\frac{1}{2}} \mathbf{u}+\beta \alpha \boldsymbol{\mu}(\rho)\right)>t\right] \mid \longrightarrow 0
\end{aligned}
$$

where

$$
\begin{aligned}
& \mathbf{A}(\rho)=\rho^{2} \frac{\left(\mathbf{H}^{*} \mathbf{Q}_{N}(\rho) \mathbf{H}\right)^{-1}}{\frac{1}{N} \operatorname{tr}\left(\mathbf{C}_{N} \mathbf{Q}_{N}(\rho)\right)} \\
& \boldsymbol{\mu}(\rho)=\frac{1}{\rho} \mathbf{H}^{*} \mathbf{Q}_{N}(\rho) \mathbf{v}
\end{aligned}
$$

$\mathbf{u}$ is a standard complex normal random vector, and $\boldsymbol{\Sigma}(\rho)$ is the asymptotic covariance matrix of the random vector $\mathbf{H}^{*} \widehat{\mathbf{R}}_{N}^{-1}(\rho) \mathbf{y}$ given by :

$$
\boldsymbol{\Sigma}(\rho)=\frac{1}{\rho^{2}} \frac{\mathbf{H}^{*} \mathbf{C}_{N} \mathbf{Q}_{N}(\rho)^{2} \mathbf{H}}{1-c m(-\rho)^{2}(1-\rho)^{2} \frac{1}{N} \operatorname{tr}\left(\mathbf{C}_{N}^{2} \mathbf{Q}_{N}(\rho)^{2}\right)}
$$

2) Compound-Gaussian clutters: As $N, n \rightarrow \infty$ with $c_{N} \rightarrow$ $c \in(0, \infty)$ and $\beta \in\{0,1\}$,

$$
\begin{aligned}
& \sup _{\rho \in \mathcal{R}_{\kappa}^{R T E}} \mid \mathbb{P}\left[\widehat{S}_{N}^{\mathrm{RTE}}(\rho)>\frac{t}{N} \mid H_{\beta}\right] \\
& -\mathbb{E}\left[\mathbb { P } \left[\left(\mathbf{u}^{*} \boldsymbol{\Sigma}\left(\underline{\rho}^{\frac{1}{2}}+\frac{\beta \alpha}{\sqrt{\tau}} \boldsymbol{\mu}(\underline{\rho})^{*}\right)\right.\right.\right. \\
& \left.\times \mathbf{A}(\underline{\rho})\left(\boldsymbol{\Sigma}\left(\underline{\rho}^{\frac{1}{2}} \mathbf{u}+\frac{\beta \alpha}{\sqrt{\tau}} \boldsymbol{\mu}(\underline{\rho})\right)>t \mid \tau\right]\right] \mid \longrightarrow 0
\end{aligned}
$$

where

$$
\begin{aligned}
\mathbf{A}(\underline{\rho}) & =\underline{\rho^{2}} \frac{\left(\mathbf{H}^{*} \mathbf{Q}_{N}(\underline{\rho}) \mathbf{H}\right)^{-1}}{\frac{1}{N} \operatorname{tr}\left(\mathbf{C}_{N} \mathbf{Q}_{N}(\underline{\rho})\right)} \\
\boldsymbol{\mu}(\underline{\rho}) & =\frac{1}{\underline{\rho}} \mathbf{H}^{*} \mathbf{Q}_{N}(\underline{\rho}) \mathbf{v},
\end{aligned}
$$

$\mathbf{u}$ is a standard complex normal random vector, and $\mathbf{\Sigma}(\underline{\rho)}$ is the asymptotic covariance matrix of the random vector $\mathbf{H}^{*} \widehat{\mathbf{R}}_{N}^{-1}(\underline{\rho}) \mathbf{y}$ given by :

$$
\boldsymbol{\Sigma}\left(\underline{\rho)}=\frac{1}{\underline{\rho}^{2}} \frac{\mathbf{H}^{*} \mathbf{C}_{N} \mathbf{Q}_{N}(\underline{\rho})^{2} \mathbf{H}}{1-c m(-\underline{\rho})^{2}(1-\underline{\rho})^{2} \frac{1}{N} \operatorname{tr}\left(\mathbf{C}_{N}^{2} \mathbf{Q}_{N}(\underline{\rho})^{2}\right)}\right.
$$




\section{Proof. See Appendix A}

Theorem 5 establishes that under $H_{0}$, the statistic $N \widehat{S}_{N}^{\mathrm{RSCM}}(\rho)$ behaves as a finite sum of exponentially distributed random variables, yielding a hypoexponential distribution. Under the null hypothesis, the expression of the asymptotic false alarm probability is given by:

$$
\begin{aligned}
& \mathbb{P}\left[\mathbf{u}^{*} \boldsymbol{\Sigma}(\rho)^{\frac{1}{2}} \mathbf{A}(\rho) \boldsymbol{\Sigma}(\rho)^{\frac{1}{2}} \mathbf{u}>t\right]= \\
& \sum_{j=1}^{r}\left(\prod_{k=1, k \neq j}^{r} \frac{\lambda_{k}(\rho)}{\lambda_{k}(\rho)-\lambda_{j}(\rho)}\right) \exp \left(-\lambda_{j}(\rho) t\right)
\end{aligned}
$$

where $\lambda_{i}(\rho), i \in\{1, \ldots, r\}$, are the eigenvalues of $(\boldsymbol{\Sigma}(\rho) \mathbf{A}(\rho))^{-1}$ assumed to be different from each other. Under $H_{1}, N \widehat{S}_{N}^{\mathrm{RSCM}}(\rho)$ behaves asymptotically as a finite sum of weighted non-central Chi-squared random variables. Such distribution does not have a closed-form expression, in contrast to the distribution obtained under $H_{0}$, and thus, its evaluation will be based on the Pearson approximation developed in [45]. Note that the same kind of distributions are obtained for $\widehat{S}_{N}^{\mathrm{RTE}}(\rho)$ under $H_{0}$ and under $H_{1}$ when conditioned on the scalar $\tau$.

The false alarm and detection probabilities in Theorem 5 come out with unknown parameters that depend on the unknown covariance matrix and therefore do not allow for an immediate tuning of the design parameters, namely the regularization parameter $\rho$ and the threshold $t$. To tackle this problem, we need to build consistent estimates of these parameters. This will be the objective of the following proposition:

Proposition 6 (Consistent estimates).1) Gaussian clutters: For $\rho \in(0,1)$, define:

$$
\begin{aligned}
\widehat{\mathbf{\Sigma}}_{R S C M}(\rho) & \triangleq \frac{\mathbf{H}^{*}\left(\frac{1}{\rho} \widehat{\mathbf{R}}_{N}^{-1}(\rho)-\widehat{\mathbf{R}}_{N}^{-2}(\rho)\right) \mathbf{H}}{\rho(1-\rho)\left(\frac{1-c}{\rho}+\frac{c}{N} \operatorname{tr} \widehat{\mathbf{R}}_{N}^{-1}(\rho)\right)^{2}} \\
\widehat{\mathbf{A}}_{R S C M}(\rho) & \triangleq \frac{\rho(1-\rho)\left(\frac{1-c}{\rho}+\frac{c}{N} \operatorname{tr} \widehat{\mathbf{R}}_{N}^{-1}(\rho)\right)}{1-\frac{\rho}{N} \operatorname{tr} \widehat{\mathbf{R}}_{N}^{-1}(\rho)} \\
& \times\left(\mathbf{H}^{*} \widehat{\mathbf{R}}_{N}^{-1}(\rho) \mathbf{H}\right)^{-1}
\end{aligned}
$$

Define as well for $\rho \in(0,1]$,

$$
\widehat{\boldsymbol{\mu}}_{R S C M}(\rho)=\mathbf{H}^{*} \widehat{\mathbf{R}}_{N}^{-1}(\rho) \mathbf{p}
$$

and let

$$
\widehat{\mathbf{A}}_{R S C M}(1) \triangleq \lim _{\rho \uparrow 1} \widehat{\mathbf{A}}_{R S C M}(\rho)=\frac{\left(\mathbf{H}^{*} \mathbf{H}\right)^{-1}}{\frac{1}{N} \operatorname{tr} \mathbf{R}_{N}}
$$

and

$$
\widehat{\boldsymbol{\Sigma}}_{R S C M}(1) \triangleq \lim _{\rho \uparrow 1} \widehat{\boldsymbol{\Sigma}}_{R S C M}(\rho)=\mathbf{H}^{*} \widehat{\mathbf{R}}_{N} \mathbf{H}
$$

Then, if $\mathbf{v}=\mathbf{p}$,

$$
\begin{aligned}
& \max \left(\sup _{\rho \in \mathcal{R}_{\kappa}^{R S C M}}\left\|\widehat{\mathbf{A}}_{R S C M}(\rho)-\mathbf{A}(\rho)\right\|,\right. \\
& \sup _{\rho \in \mathcal{R}_{\kappa}^{R S C M}}\left\|\widehat{\boldsymbol{\Sigma}}_{R S C M}(\rho)-\boldsymbol{\Sigma}(\rho)\right\|, \\
& \left.\sup _{\rho \in \mathcal{R}_{\kappa}^{R S C M}}\left\|\widehat{\boldsymbol{\mu}}_{R S C M}-\boldsymbol{\mu}(\rho)\right\|\right) \\
& \stackrel{\text { a.s. }}{\longrightarrow} 0 .
\end{aligned}
$$

2) Compound-Gaussian clutters: For $\rho \in(0,1)$, define:

$$
\begin{aligned}
& \widehat{\mathbf{\Sigma}}_{R T E}(\rho) \triangleq \frac{\left(\frac{1}{N} \operatorname{tr} \widehat{\mathbf{C}}_{N}(\rho)\right)^{2}\left(\mathbf{H}^{*}\left(\widehat{\mathbf{C}}_{N}^{-1}(\rho)-\rho \widehat{\mathbf{C}}_{N}^{-2}(\rho)\right) \mathbf{H}\right)}{\left(\frac{1}{N} \operatorname{tr} \widehat{\mathbf{C}}_{N}(\rho)-\rho\right)\left(1-c_{N}+c_{N} \rho\right)^{2}} \\
& \widehat{\mathbf{A}}_{R T E}(\rho) \triangleq \frac{(1-c+c \rho)\left(\frac{1}{N} \operatorname{tr} \widehat{\mathbf{C}}_{N}(\rho)-\rho\right)}{(1-\rho)\left(\frac{1}{N} \operatorname{tr} \widehat{\mathbf{C}}_{N}(\rho)\right)^{2}}\left(\mathbf{H}^{*} \widehat{\mathbf{C}}_{N}^{-1}(\rho) \mathbf{H}\right)^{-1}
\end{aligned}
$$

Define also for $\rho \in(0,1]$,

$$
\widehat{\boldsymbol{\mu}}_{R T E}(\rho) \triangleq\left(\frac{1}{N} \operatorname{tr} \widehat{\mathbf{C}}_{N}(\rho)\right)\left(\mathbf{H}^{*} \widehat{\mathbf{C}}_{N}^{-1}(\rho) \mathbf{p}\right)
$$

and let $\widehat{\mathbf{A}}_{R T E}(1)=\lim _{\rho \uparrow 1} \widehat{\mathbf{A}}_{R T E}(\rho)$ and $\boldsymbol{\Sigma}_{R T E}(1)=$ $\lim _{\rho \uparrow 1} \boldsymbol{\Sigma}_{R T E}(\rho)$. Then, if $\mathbf{v}=\mathbf{p}$,

$$
\begin{aligned}
& \max \left(\sup _{\rho \in \mathcal{R}_{\kappa}^{R T E}}\left\|\widehat{\mathbf{A}}_{R T E}(\rho)-\mathbf{A}(\underline{\rho})\right\|,\right. \\
& \left.\sup _{\rho \in \mathcal{R}_{\kappa}^{R T E}}\left\|\widehat{\boldsymbol{\Sigma}}_{R T E}(\rho)-\boldsymbol{\Sigma}(\underline{\rho})\right\|, \sup _{\rho \in \mathcal{R}_{\kappa}^{R T E}}\left\|\widehat{\boldsymbol{\mu}}_{R T E}(\rho)-\boldsymbol{\mu}(\underline{\rho})\right\|\right) \\
& \stackrel{\text { a.s. }}{\longrightarrow} 0 .
\end{aligned}
$$

Proof. See Appendix B.

The uniformity over $\rho$ of the errors exhibited by the estimators in Proposition 6 allows us to establish the following corollary:

Corollary 7. As $N, n \rightarrow \infty$ with $c_{N} \rightarrow c \in(0, \infty)$ and $\beta \in\{0,1\}$, we have:

$$
\begin{aligned}
& \sup _{\rho \in \mathcal{R}_{\kappa}^{R S C M}} \mid \mathbb{P}\left[\widehat{S}_{N}^{\mathrm{RSCM}}(\rho)>\frac{t}{N} \mid H_{\beta}\right] \\
& -\mathbb{P}\left[\left(\mathbf{u}^{*} \widehat{\boldsymbol{\Sigma}}_{R S C M}(\rho)^{\frac{1}{2}}+\beta \alpha \widehat{\boldsymbol{\mu}}_{R S C M}(\rho)^{*}\right) \widehat{\mathbf{A}}_{R S C M}(\rho)\right. \\
& \left.\times\left(\widehat{\boldsymbol{\Sigma}}_{R S C M}(\rho)^{\frac{1}{2}} \mathbf{u}+\beta \alpha \widehat{\boldsymbol{\mu}}_{R S C M}(\rho)\right)>t\right] \mid \longrightarrow 0
\end{aligned}
$$

and

$$
\begin{aligned}
& \sup _{\rho \in \mathcal{R}_{\kappa}^{R T E}} \mid \mathbb{P}\left[\widehat{S}_{N}^{\mathrm{RTE}}(\rho)>\frac{t}{N} \mid H_{\beta}\right] \\
& -\mathbb{E}\left[\mathbb { P } \left[\left(\mathbf{u}^{*} \widehat{\boldsymbol{\Sigma}}_{R T E}(\rho)^{\frac{1}{2}}+\frac{\beta \alpha}{\sqrt{\tau}} \widehat{\boldsymbol{\mu}}_{R T E}(\rho)^{*}\right) \widehat{\mathbf{A}}_{R T E}(\rho)\right.\right. \\
& \left.\left.\times\left(\widehat{\boldsymbol{\Sigma}}_{R T E}(\rho)^{\frac{1}{2}} \mathbf{u}+\frac{\beta \alpha}{\sqrt{\tau}} \widehat{\boldsymbol{\mu}}_{R T E}(\rho)\right)>t \mid \tau\right]\right] \mid \longrightarrow 0
\end{aligned}
$$


The result of Corollary 7 provides a consistent estimator for each $\rho$ and $t$ of the false alarm and detection probabilities. These estimates can be thus used to determine the pair of values $(\rho, t)$ that maximizes the detection probability while keeping the false alarm probability at a desired value denoted by $\eta$. This approach is optimal in regards of the considered application, but might be not be applicable in practice, as it requires the knowledge of $\alpha$ and the distribution of the scalar $\tau$ when the ASMF-RTE is considered. In order to make the selection of $\rho$ and $t$ independent of $\alpha$ and $\tau$, we propose to rather consider the suboptimal choices of $\rho, \widehat{\rho}_{R S C M}$ and $\widehat{\rho}_{R T E}$ given by:

$$
\widehat{\rho}_{R S C M}=\operatorname{argmax} \widehat{\boldsymbol{\mu}}_{R S C M}(\rho)^{*} \widehat{\boldsymbol{\Sigma}}_{R S C M}^{-1}(\rho) \widehat{\boldsymbol{\mu}}_{R S C M}(\rho)
$$

and

$$
\widehat{\rho}_{R T E}=\operatorname{argmax} \widehat{\boldsymbol{\mu}}_{R T E}(\rho)^{*} \widehat{\boldsymbol{\Sigma}}_{R T E}^{-1}(\rho) \widehat{\boldsymbol{\mu}}_{R T E}(\rho)
$$

These choices of $\rho$ can be shown to maximize an upper bound on the detection probability. To see that, consider the ASMFRSCM detector, and bound the asymptotic detection probability as in (6) where $Q_{r}$ denotes the generalized Marcum-Q function. The suboptimal value of $\rho$ is obtained by noticing that $Q_{r}$ is increasing with respect to the first argument, while its second argument in (7) is independent of $\rho$.

With the value of $\rho$ at hand, the threshold $t$ is then set to the values that tune the asymptotic false alarm probability to $\eta$, i.e,

$$
\sum_{j=1}^{r}\left(\prod_{k=1, k \neq j}^{r} \frac{\widehat{\lambda}_{k}}{\widehat{\lambda}_{k}-\widehat{\lambda}_{j}}\right) \exp \left(-\widehat{\lambda}_{j} t\right)=\eta
$$

where $\widehat{\lambda}_{j}$ 's denote the eigenvalues of
$\left(\widehat{\boldsymbol{\Sigma}}_{R S C M}\left(\widehat{\rho}_{R S C M}\right) \widehat{\mathbf{A}}_{R S C M}\left(\widehat{\rho}_{R S C M}\right)\right)^{-1}$ for the ASMFRSCM and those of $\left(\widehat{\boldsymbol{\Sigma}}_{R T E}\left(\widehat{\rho}_{R T E}\right) \widehat{\mathbf{A}}_{R T E}\left(\widehat{\rho}_{R T E}\right)\right)^{-1}$ for the RTE.

\section{NUMERICAL RESUltS}

In this section, we present some simulations in order to validate our results and to discuss some further less obvious implications. For all the experiments, we consider that the clutter covariance matrix is given by the following Toeplitz matrix:

$$
\left[\mathbf{C}_{N}\right]_{i, j}=\left\{\begin{array}{ll}
b^{j-i} & \text { if } i \leq j \\
\left(b^{i-j}\right)^{*} & \text { if } i>j
\end{array},\right.
$$

where $b$ is a scalar representing the correlation lag coefficient between two successive pulses. The temporal steering vector is given by:

$$
\mathbf{p}=\mathbf{a}\left(f_{d}\right)
$$

where $f_{d} \mapsto\left[\mathbf{a}\left(f_{d}\right)\right]_{k}=e^{\jmath 2 \pi(k-1) f_{d}}, f_{d}$ is the target Doppler frequency normalized to the radar pulse repetition frequency (PRF), $k \in 1, . ., N$, and $N$ is the number of pulses. To define an appropriate subspace that spans the actual steering vector, we follow the same approach used in [46]. In particular, we assume that $f_{d}$ is a random variable uniformly distributed in the interval $\left[f_{d_{0}}-f_{d_{\max }}, f_{d_{0}}+f_{d_{\max }}\right]$, where $f_{d_{0}}$ is the nominal Doppler frequency and $f_{d_{\max }}$ is the maximum Doppler error with $0 \leq f_{d_{\max }} \leq 0.5$. With these notations at hand, the $(i, k)$-th element of the covariance matrix $\mathbf{M}_{\mathbf{p}}$ of the vector $\mathbf{p}$ is:

$$
\left[\mathbf{M}_{\mathbf{p}}\right]_{i, k}=\operatorname{sinc}\left[2 f_{d_{\max }}(i-k)\right] e^{j 2 \pi f_{d_{0}}(i-k)},
$$

In order to determine the signal subspace, we compute the minimum number of dominant eigenvalues of $\mathbf{M}_{\mathbf{p}}$ whose sum exceeds $(1-\epsilon) \operatorname{tr}\left(\mathbf{M}_{\mathbf{p}}\right)$, where $\epsilon(0<\epsilon<1)$ is chosen close to 0 . The corresponding eigenvectors form thus the columns of matrix $\mathbf{H}$.

\section{A. ROC curves}

In these experiments, we set $f_{d_{0}}$ to $0.5, b$ to $0.95, N$ to 60 and $n$ to 120 . We consider a mismatched actual steering vector with Doppler frequency set to the median value of all possible mismatches, i.e. $f_{d}=f_{d_{0}}+\frac{f_{\max }}{2}$. We carry out Monte-Carlo simulations in order to represent the Receiver Operating Characteristics (ROC). In each trial, we generate the primary signal $\mathbf{y}=\alpha \mathbf{p}+\mathbf{x}$ and the secondary data $\mathbf{x}_{1}, \cdots, \mathbf{x}_{n}$. Based on the secondary data, the receiver estimates $\widehat{\rho}_{\mathrm{RSCM}}$ and then compute $\widehat{\mathbf{R}}_{N}\left(\widehat{\rho}_{\mathrm{RSCM}}\right)$. Finally, the threshold value is set numerically to the value that achieves the required asymptotic false alarm probability. Through a Monte Carlo averaging, we numerically compute the detection probability. We compare the detection performances of the proposed detector based on our setting of the regularization paramter with those proposed by Ledoit et al. [28] and Chen et al. [29]. Also, we compare with the non-adaptive subspace detector, in which the structure of the covariance matrix is known, in order to figure out how much loss occurs in the probability of detection due to estimation errors. Finally we compare with the Adaptive Subspace detector (ASD-SCM) [14], in which the Sample Covariance Matrix is used to estimate the covariance matrix.

The first deducted ascertainment according to Fig. 1 is that the ASMF detector outperforms the ANMF detector, for a reasonable magnitude of mismatch set to the median value. We can notice also that the loss in terms of the probability of detection of the ASMF-RSCM, with respect to the non-adaptive Subspace detector, is not large. Besides, our proposed design of the regularization parameter outperforms both designs that are proposed by Chen et al. and Ledoit et al.. The ASMFSCM scheme yields the worst performance, and this is due to the number of observations being only twofold the number of pulses $N$. Finally, the theoretical design performances considerably approach the empirical design performances shown by the Monte-Carlo simulations. Some discrepancy between theoretical and simulation results is due to the fact that the performance analysis is studied asymptotically.

The analogue experiment is repeated in the compound Gaussian clutter setting and the ROC curves of the different schemes are drawn in Fig. 2 We assume that the speckle $\tau_{i}$ has a K-Distribution with shape $\nu=0.5$. We compare with The non-adaptive subspace detector. For the RTE, we comapre with the design of $\rho$ that was proposed by Ollila et al. [30]. The same conclusions as in the Gaussian setting case are deduced. Notably, the loss with respect to the non-adaptive subspace 


$$
\begin{aligned}
& \mathbb{P}\left[\left(\mathbf{u}^{*}+\alpha \widehat{\boldsymbol{\mu}}_{R S C M}(\rho)^{*} \widehat{\boldsymbol{\Sigma}}_{R S C M}(\rho)^{-\frac{1}{2}}\right) \widehat{\boldsymbol{\Sigma}}_{R S C M}(\rho)^{\frac{1}{2}} \widehat{\mathbf{A}}_{R S C M}(\rho) \widehat{\boldsymbol{\Sigma}}_{R S C M}(\rho)^{\frac{1}{2}}\left(\mathbf{u}+\alpha \widehat{\boldsymbol{\Sigma}}_{R S C M}(\rho)^{-\frac{1}{2}} \widehat{\boldsymbol{\mu}}_{R S C M}(\rho)\right)>t\right] \\
& \leq \mathbb{P}\left[\left\|\sqrt{2} \mathbf{u}+\alpha \sqrt{2} \widehat{\boldsymbol{\Sigma}}_{R S C M}(\rho)^{-\frac{1}{2}} \widehat{\boldsymbol{\mu}}_{R S C M}(\rho)\right\|^{2}>\frac{2 t}{\sup _{\rho \in \mathcal{R}_{\kappa}^{R S C M}}\left\|\widehat{\boldsymbol{\Sigma}}_{R S C M}(\rho)^{\frac{1}{2}} \widehat{\mathbf{A}}_{R S C M}(\rho) \widehat{\boldsymbol{\Sigma}}_{R S C M}(\rho)^{\frac{1}{2} \|}\right\|}\right] \\
& =Q_{r}\left(\sqrt{2 \widehat{\boldsymbol{\mu}}_{R S C M}(\rho)^{*} \widehat{\boldsymbol{\Sigma}}_{R S C M}^{-1}(\rho) \widehat{\boldsymbol{\mu}}_{R S C M}(\rho)}, \sqrt[t]{\frac{t}{\sup _{\rho \in \mathcal{R}_{\kappa}^{R S C M}}\left\|\widehat{\boldsymbol{\Sigma}}_{R S C M}(\rho)^{\frac{1}{2}} \widehat{\mathbf{A}}_{R S C M}(\rho) \widehat{\boldsymbol{\Sigma}}_{R S C M}(\rho)^{\frac{1}{2}}\right\|}}\right)
\end{aligned}
$$

detector is not large, which illustrates the robustness the RTE for our proposed design of $\rho$. Finally, our optimized $\rho$ that is set for the RTE yields better performance than the $\rho$ designed by Ollila et al..

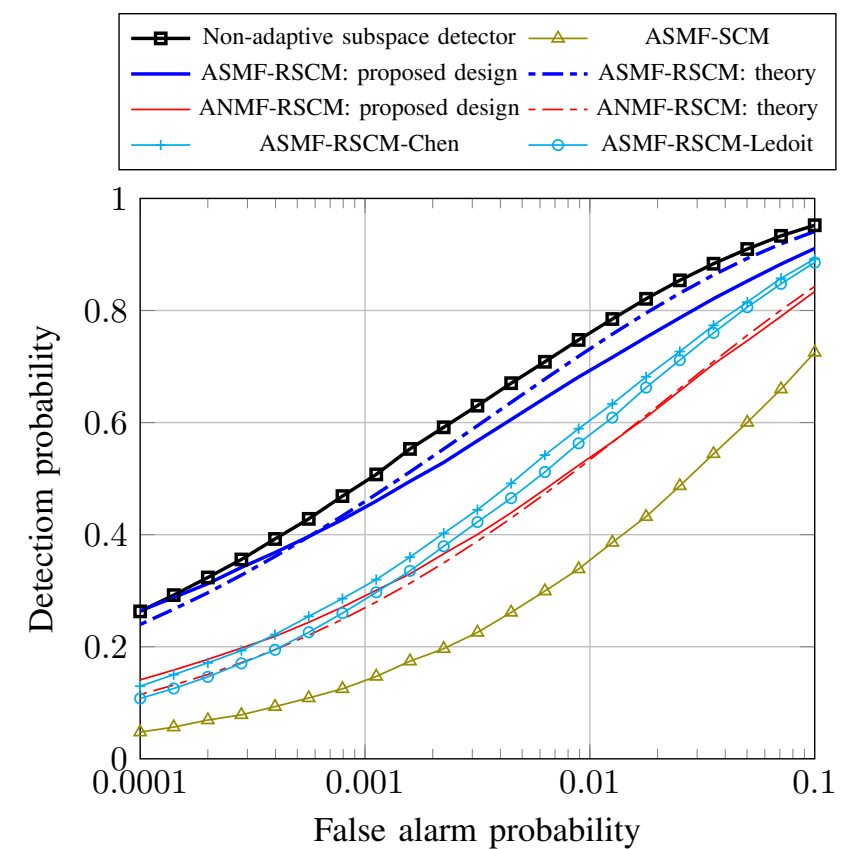

Fig. 1. ROC curves for $\alpha=0.5, b=0.95, f_{d_{0}}=0.5, f_{d_{\max }}=0.015$, $f_{d}=f_{d_{0}}-\frac{f_{d_{\max }}}{2}, N=60, n=120$ and $\epsilon=10^{-3}$ so that $r=3$, Gaussian setting

\section{B. Mismatch effect on the detection probability}

This section is devoted to investigate the impact of the mismatch magnitude on both the ASMF detector and the ANMF detector. This can illustrate the best detector among the latter and ultimately nail down the critical mismatch magnitude yielding the same performance in terms of the detection probability for both detectors. Fig. 3 and Fig. 4 show the effect of the mismatch magnitude on the detection probability. We can notice that the mismatch axis can be spilt into two areas, the first area is where the ANMF detector outperforms the ASMF detector, $0<\left|f_{d}-f_{d_{0}}\right|<f_{c}$, and the second area is where the ASMF detector outperforms the ANMF detector, $f_{c}<\left|f_{d}-f_{d_{0}}\right|<f_{d_{\max }}$. In fact, It is expectable that for small mismatch magnitudes the ANMF would have

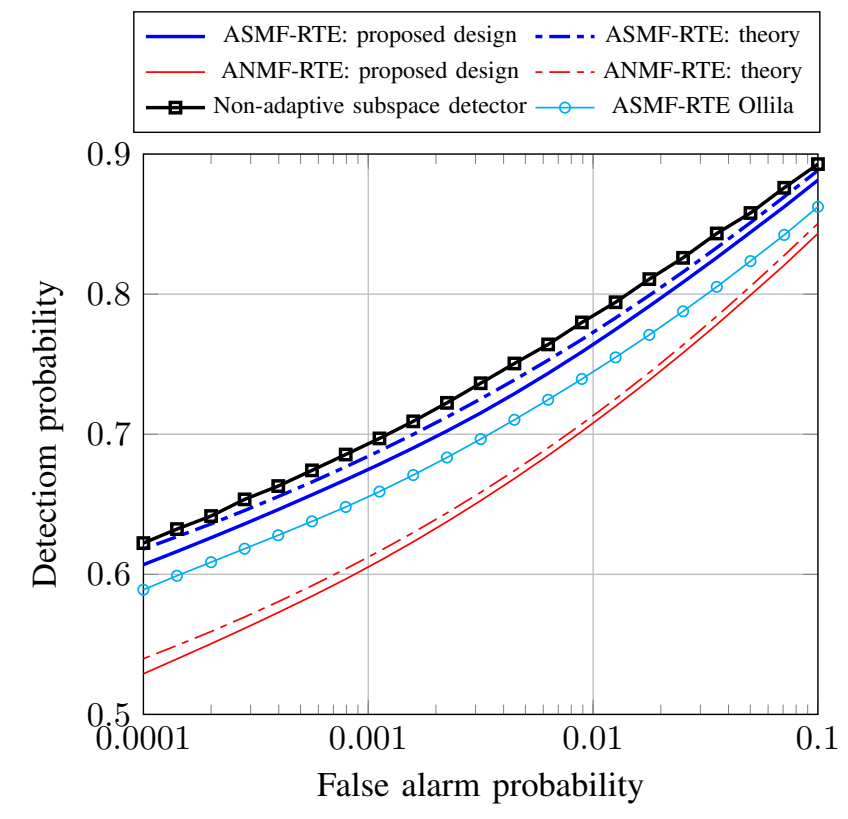

Fig. 2. ROC curves for $\alpha=0.5, b=0.95, f_{d_{0}}=0.5, f_{d_{\max }}=0.015$, $f_{d}=f_{d_{0}}-\frac{f_{d_{\max }}}{2}, N=60, n=120$ and $\epsilon=10^{-3}$ so that $r=3$,
K-distributed clutter

better performance than the ASMF, since the nominal steering vector in this case is very close to the actual one. However, for higher mismatch magnitudes, the detection probability of the ANMF vanishes dramatically whilst the ASMF keeps the same detection capability.Finally, different values of the SNR are considered and no dependency between critical mismatch magnitude and the SNR is shown by simulations., which possibly imply that increasing the SNR does not reduce the mismatch severity for the ANMF detectors.

\section{CONCLUSION}

In this paper, we investigated the problem of designing ASMF detectors. The RSCM and the RTE are used in the SMF detector statistic as a replacement of the unknown covariance matrix, thereby yielding the schemes ASMF-RSCM and ASMF-RTE. One major bottleneck toward determining the regularization parameter that optimizes the performances of ASMF detectors is linked to the difficulty to clearly characterize the distribution of the ASMF statistics under the cases of presence or absence of a signal of interest. In order to deal with this issue, we considered the regime in which the 


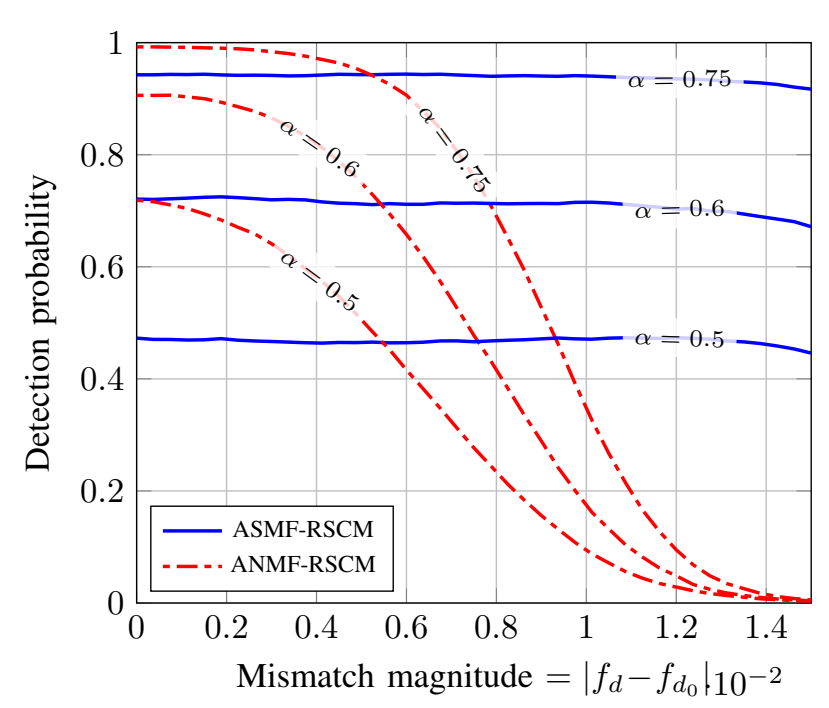

Fig. 3. Effect of the mismatch on the probability of detection for $\alpha=0.5,0.6$ and $0.75, b=0.95$, false alarm probability $=10^{-3}, f_{d_{0}}=0.5 f_{d_{\max }}=$ $0.015, N=60, n=120, \epsilon=10^{-3}$ and $r=3$, Gaussian setting

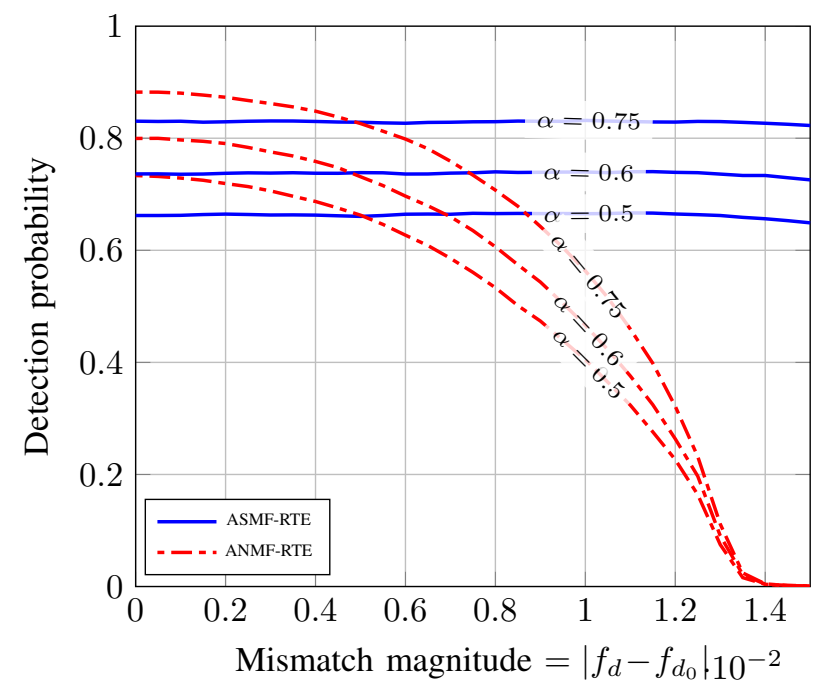

Fig. 4. Effect of the mismatch on the probability of detection for $\alpha=0.5,0.6$ and $0.75, b=0.95$, false alarm probability $=10^{-3}, f_{d_{0}}=0.5 f_{d_{\max }}=$ 0.015, $N=60, n=120$ and $\epsilon=10^{-3}$ and $r=3$, K-distributed clutter

number of samples and their dimensions grow large simultaneously. Based on tools from random matrix theory along with recent asymptotic results on the behaviour of the RTE, we analysed the asymptotic behavior of ASMF test statistics under hypotheses $H_{0}$ and $H_{1}$. The obtained results have allowed us to propose an optimal design of the regularization parameter that maximizes the detection probability while keeping fixed the false alarm probability through an appropriate tuning of the threshold value. Simulation results clearly illustrate the gain of ASMF detectors over ANMF detectors, even for small magnitudes of mismatch, and also show that the setting of the regularization parameter is better than other proposed settings in the literature.
APPENDIX A

\section{Proof of THEOREM 5}

1) In order to prove the first item of Theorem 5, we start by writing $\widehat{S}_{N}^{\mathrm{RSCM}}$ as:

$$
N \widehat{S}_{N}^{\mathrm{RSCM}}=\frac{\left\|\left(\mathbf{H}^{*} \widehat{\mathbf{R}}_{N}^{-1}(\rho) \mathbf{H}\right)^{-\frac{1}{2}} \mathbf{H}^{*} \widehat{\mathbf{R}}_{N}^{-1}(\rho) \mathbf{y}\right\|^{2}}{\frac{1}{N} \mathbf{y}^{*} \widehat{\mathbf{R}}_{N}^{-1}(\rho) \mathbf{y}}
$$

Using standard results from random matrix theory, we know that for all $\rho$ in $[\kappa, 1]$,

$$
\begin{gathered}
\mathbf{H}^{*} \widehat{\mathbf{R}}_{N}^{-1}(\rho) \mathbf{y}=\mathcal{O}_{p}(1), \\
\mathbf{H}^{*} \widehat{\mathbf{R}}_{N}^{-1}(\rho) \mathbf{H}-\frac{1}{\rho} \mathbf{H}^{*} \mathbf{Q}(\rho) \mathbf{H} \stackrel{\text { a.s. }}{\longrightarrow} 0 .
\end{gathered}
$$

and

$$
\frac{1}{N} \mathbf{y}^{*} \widehat{\mathbf{R}}_{N}^{-1}(\rho) \mathbf{y}-\frac{1}{N \rho} \operatorname{tr} \mathbf{C}_{N} \mathbf{Q}(\rho) \stackrel{\text { a.s. }}{\longrightarrow} 0 .
$$

Hence, using the fact that $\lambda_{\min }\left(\mathbf{H}^{*} \mathbf{H}\right)$ is bounded away zero for $n$ large enough, we obtain:

$$
\begin{aligned}
N \widehat{S}_{N}^{\mathrm{RSCM}} & =\frac{\rho^{2}\left\|\left(\mathbf{H}^{*} \mathbf{Q}(\rho) \mathbf{H}\right)^{-\frac{1}{2}} \mathbf{H}^{*} \widehat{\mathbf{R}}_{N}^{-1}(\rho) \mathbf{y}\right\|^{2}}{\frac{1}{N} \operatorname{tr} \mathbf{C}_{N} \mathbf{Q}(\rho)}+o_{p}(1) \\
& =\left\|\widetilde{\mathbf{H}}^{*} \widehat{\mathbf{R}}_{N}^{-1}(\rho) \mathbf{y}\right\|^{2}+o_{p}(1)
\end{aligned}
$$

where $\widetilde{\mathbf{H}}=\mathbf{H A}^{\frac{1}{2}}(\rho)$ with $\mathbf{A}(\rho)$ being given by (4).

The second step is to show that the vector $\widetilde{\mathbf{H}}^{*} \widetilde{\mathbf{R}}_{N}^{-1}(\rho) \mathbf{y}$ is asymptotically well approximated by a Gaussian vector with a given mean and covariance matrix. The proof relies on computing an asymptotic expression for the characteristic function of $\left[\begin{array}{l}\Re\left(\widetilde{\mathbf{H}}^{*} \widehat{\mathbf{R}}_{N}^{-1}(\rho) \mathbf{y}\right) \\ \Im\left(\widetilde{\mathbf{H}}^{*} \widehat{\mathbf{R}}_{N}^{-1}(\rho) \mathbf{y}\right)\end{array}\right]$ for a given argument $\mathbf{t} \in \mathbb{R}^{2 r}$. To this end, let $\widetilde{\mathbf{H}}=\mathbf{H A}^{\frac{1}{2}}(\rho)$ and $\mathbf{G}=\left[\begin{array}{ll}\mathbf{y} & \widetilde{\mathbf{H}}\end{array}\right] \in \mathbb{C}^{N \times(r+1)}$. From [47, Lemma 5.3] (adapted to our current notations and normalizations), for any Hermitian $\mathbf{B} \in \mathbb{C}^{(r+1) \times(r+1)}$

$$
\begin{aligned}
& \mathbb{E}\left[\exp \left(\imath \operatorname{tr} \mathbf{B G}^{*}\left[\widehat{\mathbf{R}}_{N}^{-1}(\rho)-\frac{1}{\rho} \mathbf{Q}_{N}(\rho)\right] \mathbf{G}\right) \mid \mathbf{y}\right] \\
& =\exp \left(-\frac{1}{2} \Delta_{N}^{2}(\mathbf{B} ; \mathbf{y} ; \mathbf{H})\right)+O\left(N^{-\frac{1}{2}}\right)
\end{aligned}
$$

where we denote by $\mathbb{E}[\cdot \mid y]$ the conditional expectation with respect to the random vector $\mathbf{y}$ and where:

$$
\Delta_{N}^{2}(\mathbf{B} ; \mathbf{y} ; \mathbf{H}) \triangleq \frac{c m_{N}(-\rho)^{2}(1-\rho)^{2} \operatorname{tr}\left(\mathbf{G} \mathbf{B} \mathbf{G}^{*} \mathbf{C}_{N} \mathbf{Q}_{N}(\rho)^{2}\right)^{2}}{\rho^{2}\left(1-c m_{N}(-\rho)^{2}(1-\rho)^{2} \frac{1}{N} \operatorname{tr} \mathbf{C}_{N}^{2} \mathbf{Q}_{N}(\rho)^{2}\right)}
$$

We set matrix $\mathbf{B}$ to:

$$
\mathbf{B}=\left[\begin{array}{cc}
0 & \mathbf{b}^{*} \\
\mathbf{b} & \mathbf{0}_{r \times r}
\end{array}\right]
$$

where $\mathbf{b}=\left[b_{1}, \cdots, b_{r}\right]^{T}$ is some $\mathbb{C}^{r \times 1}$ vector. Thus, simple calculations lead to:

$$
\begin{aligned}
& \operatorname{tr} \mathbf{B} \frac{1}{\rho} \mathbf{G}^{*} \mathbf{Q}_{N}(\rho) \mathbf{G}=\frac{1}{\rho} \sum_{i=1}^{r} b_{i}^{*} \widetilde{\mathbf{h}}_{i}^{*} \mathbf{Q}_{N}(\rho) \mathbf{y}+b_{i} \mathbf{y}^{*} \mathbf{Q}_{N}(\rho) \widetilde{\mathbf{h}}_{i} \\
& =\frac{1}{\rho}\left[\begin{array}{ll}
\mathbf{b}^{*} & \mathbf{b}^{T}
\end{array}\right]\left[\begin{array}{c}
\widetilde{\mathbf{H}}^{*} \mathbf{Q}_{N}(\rho) \mathbf{y} \\
\left(\mathbf{y}^{*} \mathbf{Q}_{N}(\rho) \widetilde{\mathbf{H}}\right)^{T}
\end{array}\right],
\end{aligned}
$$


where $\widetilde{\mathbf{h}}_{i}$ denote the $i$-th column of matrix $\widetilde{\mathbf{H}}$. In the same way, we also have:

$$
\operatorname{tr} \mathbf{B G}^{*} \widehat{\mathbf{R}}_{N}^{-1}(\rho) \mathbf{G}=\left[\begin{array}{ll}
\mathbf{b}^{*} & \mathbf{b}^{T}
\end{array}\right]\left[\begin{array}{c}
\widetilde{\mathbf{H}}^{*} \widehat{\mathbf{R}}_{N}^{-1}(\rho) \mathbf{y} \\
\left(\mathbf{y}^{*} \widehat{\mathbf{R}}_{N}^{-1}(\rho) \widetilde{\mathbf{H}}\right)^{T}
\end{array}\right]
$$

Furthermore, note that $\frac{1}{\rho}\left[\begin{array}{c}\widetilde{\mathbf{H}}^{*} \mathbf{Q}_{N}(\rho) \mathbf{y} \\ \left(\mathbf{y}^{*} \mathbf{Q}_{N}(\rho) \widetilde{\mathbf{H}}\right)^{T}\end{array}\right]$ is a Gaussian vector with mean $\beta \alpha\left[\begin{array}{c}\mathbf{A}^{\frac{1}{2}}(\rho) \boldsymbol{\mu}(\rho) \\ \left(\boldsymbol{\mu}(\rho)^{*} \mathbf{A}^{\frac{1}{2}}(\rho)\right)^{T}\end{array}\right]$ and covariance matrix:

$$
\frac{1}{\rho^{2}}\left[\begin{array}{cc}
\widetilde{\mathbf{H}}^{*} \mathbf{Q}^{2}(\rho) \mathbf{C}_{N} \widetilde{\mathbf{H}} & \mathbf{0}_{r \times r} \\
\mathbf{0}_{r \times r} & \left(\widetilde{\mathbf{H}}^{*} \mathbf{Q}^{2}(\rho) \mathbf{C}_{N} \widetilde{\mathbf{H}}\right)^{T}
\end{array}\right]
$$

Hence, $\operatorname{tr} \mathbf{B}\left[\frac{1}{\rho} \mathbf{G}^{*} \mathbf{Q}_{N}(\rho) \mathbf{G}\right]$ is a Gaussian random variable with mean

$$
\nu_{N}(\rho) \triangleq \beta \alpha \mathbf{b}^{*} \mathbf{A}^{\frac{1}{2}} \boldsymbol{\mu}(\rho)+\beta \alpha \mathbf{b}^{T}\left(\boldsymbol{\mu}^{*}(\rho) \mathbf{A}^{\frac{1}{2}}(\rho)\right)^{T}
$$

and variance:

$$
\Delta_{N}^{\prime 2}(\mathbf{B} ; \mathbf{H}) \triangleq \frac{2}{\rho^{2}} \mathbf{b}^{*}\left(\widetilde{\mathbf{H}}^{*} \mathbf{C}_{N} \mathbf{Q}_{N}(\rho)^{2} \widetilde{\mathbf{H}}\right) \mathbf{b} .
$$

Consequently, we conclude:

$$
\begin{aligned}
& \mathbb{E}\left[\exp \left(\imath \operatorname{tr} \mathbf{B}\left[\frac{1}{\rho} \mathbf{G}^{*} \mathbf{Q}_{N}(\rho) \mathbf{G}\right]\right)\right] \\
& =\exp \left(-\frac{1}{2} \Delta_{N}^{\prime 2}(\mathbf{B} ; \mathbf{H})\right) \exp \left(\imath \nu_{N}(\rho)\right)+O\left(N^{-\frac{1}{2}}\right)
\end{aligned}
$$

With this result at hand, we are now in position to remove the conditioning on $\mathbf{y}$ in 10$]$. We have:

$$
\begin{aligned}
& \mathbb{E}\left[\exp \left(\imath \sqrt{N} \operatorname{tr} \mathbf{B}\left[\mathbf{G}^{*} \widehat{\mathbf{R}}_{N}^{-1}(\rho) \mathbf{G}\right]\right)\right] \\
& =\mathbb{E}_{\mathbf{y}}\left[\exp \left(-\frac{1}{2} \Delta_{N}^{2}(\mathbf{B} ; \mathbf{y} ; \mathbf{H})\right) \exp \left(\imath \operatorname{tr} \mathbf{B G}^{*} \frac{1}{\rho} \mathbf{Q}_{N}(\rho) \mathbf{G}\right)\right] \\
& +O\left(N^{-\frac{1}{2}}\right) .
\end{aligned}
$$

where $\mathbb{E}_{\mathbf{y}}$ refers to the expectation with respect to the distribution of $\mathbf{y}$. Note now that

$$
\mathbf{G}^{*} \mathbf{C}_{N} \mathbf{Q}_{N}(\rho)^{2} \mathbf{G}-\mathbf{\Upsilon}_{N} \stackrel{\text { a.s. }}{\longrightarrow} 0
$$

where

$$
\mathbf{\Upsilon}_{N} \triangleq\left[\begin{array}{cc}
\frac{1}{N} \operatorname{tr} \mathbf{C}_{N}^{2} \mathbf{Q}_{N}(\rho)^{2} & 0 \\
0 & \widetilde{\mathbf{H}}^{*} \mathbf{C}_{N} \mathbf{Q}_{N}(\rho)^{2} \widetilde{\mathbf{H}}
\end{array}\right]
$$

Hence, using the dominated convergence along with (11), we obtain:

$$
\begin{aligned}
& \mathbb{E}\left[\exp \left(\imath \operatorname{tr} \mathbf{B}\left[\mathbf{G}^{*} \widehat{\mathbf{R}}_{N}^{-1}(\rho) \mathbf{G}\right]\right)\right] \\
& =\exp \left(-\frac{1}{2}\left[\Delta_{N}^{2}(\mathbf{B} ; \mathbf{H})+\Delta_{N}^{\prime 2}(\mathbf{B} ; \mathbf{H})\right]\right) \exp \left(\imath \nu_{N}(\rho)\right)+o(1)
\end{aligned}
$$

where we defined

$\Delta_{N}^{2}(\mathbf{B} ; \mathbf{H}) \triangleq \frac{c m_{N}(-\rho)^{2}(1-\rho)^{2} \operatorname{tr}\left(\mathbf{B} \boldsymbol{\Upsilon}_{N}\right)^{2}}{\rho^{2}\left(1-c m_{N}(-\rho)^{2}(1-\rho)^{2} \frac{1}{N} \operatorname{tr} \mathbf{C}_{N}^{2} \mathbf{Q}^{2}(\rho)\right)}$.
We can furthermore write

$$
\operatorname{tr}\left(\mathbf{B} \boldsymbol{\Upsilon}_{N}\right)^{2}=\frac{2}{N} \operatorname{tr}\left(\mathbf{C}_{N}^{2} \mathbf{Q}^{2}(\rho)\right) \mathbf{b}^{*} \widetilde{\mathbf{H}}^{*} \mathbf{C}_{N} \mathbf{Q}^{2}(\rho) \widetilde{\mathbf{H}} \mathbf{b} .
$$

Therefore,

$$
\begin{aligned}
\Delta_{N}^{2}(\mathbf{B} ; \mathbf{H}) & =\frac{c m_{N}(-\rho)^{2}(1-\rho)^{2} \frac{2}{N} \operatorname{tr}\left(\mathbf{C}_{N}^{2} \mathbf{Q}^{2}(\rho)\right)}{\rho^{2}\left(1-c m_{N}(-\rho)^{2}(1-\rho)^{2} \frac{1}{N} \operatorname{tr} \mathbf{C}_{N}^{2} \mathbf{Q}^{2}(\rho)\right)} \\
& \times \mathbf{b}^{*} \widetilde{\mathbf{H}}^{*} \mathbf{C}_{N} \mathbf{Q}^{2}(\rho) \widetilde{\mathbf{H}} \mathbf{b},
\end{aligned}
$$

and thus:

$$
\begin{aligned}
& \Delta_{N}^{2}(\mathbf{B} ; \mathbf{H})+\Delta_{N}^{\prime 2}(\mathbf{B} ; \mathbf{H}) \\
& =\frac{2 \mathbf{b}^{*} \widetilde{\mathbf{H}}^{*} \mathbf{C}_{N} \mathbf{Q}_{N}^{2}(\rho) \widetilde{\mathbf{H}} \mathbf{b}}{\rho^{2}\left(1-c m_{N}(-\rho)^{2}(1-\rho)^{2} \frac{1}{N} \operatorname{tr} \mathbf{C}_{N}^{2} \mathbf{Q}_{N}(\rho)^{2}\right)}
\end{aligned}
$$

Decompose $\mathbf{t}$ as $\mathbf{t}=\left[\begin{array}{l}\mathbf{t}_{1} \\ \mathbf{t}_{2}\end{array}\right]$ where $\mathbf{t}_{1}$ and $\mathbf{t}_{2}$ are in $\mathbb{R}^{r}$ and consider $\mathbf{b}=\frac{\mathbf{t}_{1}}{2}+\frac{\imath \mathbf{t}_{2}}{2}$. Then,

$$
\operatorname{tr} \mathbf{B} \mathbf{G}^{*} \widehat{\mathbf{R}}_{N}^{-1}(\rho) \mathbf{G}=\left[\begin{array}{ll}
\mathbf{t}_{1}^{T} & \mathbf{t}_{2}^{T}
\end{array}\right]\left[\begin{array}{l}
\Re\left(\widetilde{\mathbf{H}}^{*} \widehat{\mathbf{R}}_{N}^{-1}(\rho) \mathbf{y}\right) \\
\Im\left(\widetilde{\mathbf{H}}^{*} \widehat{\mathbf{R}}_{N}^{-1}(\rho) \mathbf{y}\right)
\end{array}\right]
$$

Substituting $\mathbf{b}$ by $\frac{1}{2} \mathbf{t}_{1}+\imath \frac{1}{2} \mathbf{t}_{2}$ in (12), we finally get:

$$
\begin{aligned}
& \mathbb{E}\left[\exp \left(\imath \mathbf{t}_{1}^{T} \Re\left(\widetilde{\mathbf{H}}^{*} \widehat{\mathbf{R}}_{N}^{-1}(\rho) \mathbf{y}\right)+\imath \mathbf{t}_{2}^{T} \Im\left(\widetilde{\mathbf{H}}^{*} \widehat{\mathbf{R}}_{N}^{-1}(\rho) \mathbf{y}\right)\right)\right] \\
& =\exp \left(-\frac{1}{2}\left[\mathbf{t}_{1}^{T} \mathbf{t}_{2}^{T}\right] \boldsymbol{\Phi}\left[\begin{array}{l}
\mathbf{t}_{1} \\
\mathbf{t}_{2}
\end{array}\right]\right. \\
& \left.+\imath \beta\left[\begin{array}{ll}
\mathbf{t}_{1}^{T} & \mathbf{t}_{2}^{T}
\end{array}\right]\left[\begin{array}{l}
\Re\left(\mathbf{A}^{\frac{1}{2}}(\rho) \alpha \boldsymbol{\mu}(\rho)\right) \\
\Im\left(\mathbf{A}^{\frac{1}{2}}(\rho) \alpha \boldsymbol{\mu}(\rho)\right)
\end{array}\right]\right)+o_{p}(1)
\end{aligned}
$$

where $\boldsymbol{\Phi}$ is given by:

$$
\boldsymbol{\Phi}=\left[\begin{array}{lc}
\frac{1}{2} \Re\left(\mathbf{A}(\rho)^{\frac{1}{2}} \boldsymbol{\Sigma}(\rho) \mathbf{A}(\rho)^{\frac{1}{2}}\right) & -\frac{1}{2} \Im\left(\mathbf{A}(\rho)^{\frac{1}{2}} \boldsymbol{\Sigma}(\rho) \mathbf{A}(\rho)^{\frac{1}{2}}\right) \\
\frac{1}{2} \Im\left(\mathbf{A}(\rho)^{\frac{1}{2}} \boldsymbol{\Sigma}(\rho) \mathbf{A}(\rho)^{\frac{1}{2}}\right) & \frac{1}{2} \Re\left(\mathbf{A}(\rho)^{\frac{1}{2}} \boldsymbol{\Sigma}(\rho) \mathbf{A}(\rho)^{\frac{1}{2}}\right)
\end{array}\right]
$$

Hence, using the Cramer-Wold device as in [35], we get that:

$$
\widetilde{\mathbf{H}}^{*} \widehat{\mathbf{R}}_{N}^{-1}(\rho) \mathbf{y}=\beta \alpha \mathbf{A}^{\frac{1}{2}}(\rho) \boldsymbol{\mu}(\rho)+\mathbf{A}^{\frac{1}{2}}(\rho) \boldsymbol{\Sigma}^{\frac{1}{2}}(\rho) \mathbf{z}+o_{p}(1)
$$

where $\mathbf{z} \sim \mathcal{C} \mathcal{N}\left(0, \mathbf{I}_{r}\right)$. For a fixed $\rho$, the false alarm and detection probabilities can be derived by resorting to the uniform continuous mapping theorem as in [36. Proof of Theorem 3], whereas the uniformity over $\rho$ can be obtained along the same lines as in [35]. Details are thus omitted.

2) We move now to the determination of the fluctuations of $\widehat{S}_{N}^{\mathrm{RTE}}(\rho)$. Using proposition 2, we can prove that $\widehat{S}_{N}^{R T E}$ has the same fluctuations as $S_{N}^{R T E}$ obtained by replacing $\widehat{\mathbf{C}}_{N}(\rho)$ by $\widehat{\mathbf{S}}_{N}(\rho)$ given by $(2)$ :

$$
\widetilde{S}_{N}^{R T E}=\frac{\mathbf{y}^{*} \widehat{\mathbf{S}}_{N}^{-1}(\rho) \mathbf{H}\left(\mathbf{H}^{*} \widehat{\mathbf{S}}_{N}^{-1}(\rho) \mathbf{H}\right)^{-1} \mathbf{H}^{*} \widehat{\mathbf{S}}_{N}^{-1}(\rho) \mathbf{y}}{\mathbf{y}^{*} \widehat{\mathbf{S}}_{N}^{-1}(\rho) \mathbf{y}}
$$

Since $\widetilde{S}_{N}^{R T E}$ remains unchanged after scaling $\widehat{\mathbf{S}}_{N}(\rho)$ and y, we also have:

$\widetilde{S}_{N}^{R T E}=\frac{\frac{1}{\sqrt{\tau}} \mathbf{y}^{*} \widehat{\mathbf{S}}_{N}^{-1}(\rho) \mathbf{H}\left(\mathbf{H}^{*} \widehat{\mathbf{S}}_{N}^{-1}(\rho) \mathbf{H}\right)^{-1} \frac{1}{\sqrt{\tau}} \mathbf{H}^{*} \widehat{\mathbf{S}}_{N}^{-1}(\rho) \mathbf{y}}{\frac{1}{\tau} \mathbf{y}^{*} \widehat{\mathbf{S}}_{N}^{-1}(\rho) \mathbf{y}}$ 
where $\tau=1$ under $H_{0}$. It turns out that conditionally to $\tau$. the fluctuations of $\widetilde{S}_{N}^{R T E}$ are the same as those obtained previously for $\widetilde{S}_{N}^{R S C M}$ with the single difference that $\alpha$ is replaced by $\frac{\alpha}{\sqrt{\tau}}$ and $\rho$ by $\underline{\rho}$. This ends the proof of the second item of Theorem 5 .

\section{APPENDIX B}

\section{PROOF OF PROPOSITION 6}

We will only prove the consistency of the estimators $\widehat{\boldsymbol{\Sigma}}_{R S C M}(\rho)$ and $\widehat{\boldsymbol{\Sigma}}_{R T E}(\rho)$. The handling of the other terms in proposition 6 can be carried out similarly.

We start by analyzing the consistency of $\widehat{\boldsymbol{\Sigma}}_{R S C M}(\rho)$. To this end, similarly to [36], consider the set $\mathcal{R}_{\kappa}^{\prime} \triangleq[\kappa, 1-\kappa]$. For $\rho \in \mathcal{R}_{\kappa}^{\prime}$, notice that:

$$
\begin{aligned}
& \frac{d}{d z}\left[\mathbf{H}^{*}\left(-z\left[\mathbf{I}_{N}+(1-\rho) m_{N}(z) \mathbf{C}_{N}\right]\right)^{-1} \mathbf{H}\right]_{z=-\rho} \\
& =\frac{1}{\rho^{2}} \mathbf{H}^{*} \mathbf{Q}_{N}(\rho) \mathbf{H}-\frac{1}{\rho}(1-\rho) m_{N}^{\prime}(-\rho) \mathbf{H}^{*} \mathbf{C}_{N} \mathbf{Q}_{N}(\rho)^{2} \mathbf{H} \\
& =\frac{1}{\rho^{2}} \mathbf{H}^{*} \mathbf{Q}_{N}(\rho) \mathbf{H}-\frac{1}{\rho} \frac{(1-\rho) m_{N}^{2}(-\rho) \mathbf{H}^{*} \mathbf{C}_{N} \mathbf{Q}_{N}(\rho)^{2} \mathbf{H}}{1-c m_{N}(-\rho)^{2}(1-\rho)^{2} \frac{1}{N} \operatorname{tr} \mathbf{C}_{N}^{2} \mathbf{Q}_{N}(\rho)^{2}} \\
& =\frac{1}{\rho^{2}} \mathbf{H}^{*} \mathbf{Q}_{N}(\rho) \mathbf{H}-\rho(1-\rho) m_{N}(-\rho)^{2} \mathbf{\Sigma}_{N}(\rho) .
\end{aligned}
$$

Hence, $\boldsymbol{\Sigma}_{N}(\rho)$ can be alternatively written as:

$$
\boldsymbol{\Sigma}_{N}(\rho)=\frac{\frac{1}{\rho^{2}} \mathbf{H}^{*} \mathbf{Q}_{N}(\rho) \mathbf{H}}{m_{N}(-\rho)^{2} \rho(1-\rho)}+\frac{\left[\frac{d}{d z} \frac{1}{z} \mathbf{H}^{*} \mathbf{Q}(-z) \mathbf{H}\right]_{z=-\rho}}{m_{N}(-\rho)^{2} \rho(1-\rho)}
$$

With this decomposition of $\boldsymbol{\Sigma}_{N}(\rho)$ at hand, it suffices to determine a consistent estimate for each of $\mathbf{H}^{*} \mathbf{Q}_{N}(\rho) \mathbf{H}, m_{N}(-\rho)$ and $\left[\frac{d}{d z} \frac{1}{z} \mathbf{H}^{*} \mathbf{Q}(-z) \mathbf{H}\right]_{z=-\rho}$. For this, we shall recall the following results. Let $z \in \mathbb{C} \backslash \mathbb{R}^{+}$and define:

$$
\widehat{\widehat{\mathbf{R}}}_{N}(z) \triangleq(1-\rho) \frac{1}{n} \sum_{i=1}^{n} \mathbf{z}_{i} \mathbf{z}_{i}^{*}-z \mathbf{I}_{N}
$$

(so in particular $\underline{\widehat{\mathbf{R}}}_{N}(-\rho)=\widehat{\mathbf{R}}_{N}(\rho)$, for all $\underline{\rho} \in \mathcal{R}_{\kappa}$ ), we have, with $\mathcal{C}$ a compact set of $\mathbb{C} \backslash \mathbb{R}^{+}$and any integer $k$,

$$
\begin{aligned}
& \sup _{\bar{z} \in \mathcal{C}} \| \frac{d^{k}}{d z^{k}}\left\{\mathbf{H}^{*} \widehat{\mathbf{R}}_{N}(z)^{-1} \mathbf{H}\right. \\
& \left.-\mathbf{H}^{*}\left(-z\left[\mathbf{I}_{N}+(1-\rho) m_{N}(z) \mathbf{C}_{N}\right]\right)^{-1} \mathbf{H}\right\}_{z=\bar{z}} \| \stackrel{\text { a.s. }}{\longrightarrow} 0
\end{aligned}
$$

Hence,

$$
\sup _{\rho \in \mathcal{R}_{\kappa}^{\prime}}\left\|\frac{1}{\rho} \mathbf{H}^{*} \mathbf{Q}_{N}(\rho) \mathbf{H}-\mathbf{H}^{*} \widehat{\mathbf{R}}_{N}^{-1}(\rho) \mathbf{H}\right\| \stackrel{\text { a.s. }}{\longrightarrow} 0,
$$

and

$$
\sup _{\rho \in \mathcal{R}_{\kappa}^{\prime}}\left\|\mathbf{H}^{*} \widehat{\mathbf{R}}_{N}^{-2}(\rho) \mathbf{H}+\left[\frac{d}{d z} \frac{1}{z} \mathbf{H}^{*} \mathbf{Q}(-z) \mathbf{H}\right]_{z=-\rho}\right\| \stackrel{\text { a.ss }}{\longrightarrow} 0 .
$$

Recall also from [36] that a consistent estimate of $m_{N}(-\rho)$ is given by $\frac{1-c_{N}}{\rho}+\frac{c_{N}}{N} \operatorname{tr} \widehat{\mathbf{R}}_{N}^{-1}(\rho)$ in the sense that:

$$
\sup _{\rho \in \mathcal{R}_{\kappa}^{\prime}}\left|m_{N}(-\rho)-\left(\frac{1-c_{N}}{\rho}+\frac{c_{N}}{N} \operatorname{tr} \widehat{\mathbf{R}}_{N}^{-1}(\rho)\right)\right| \stackrel{\text { a.s. }}{\longrightarrow} 0 .
$$

Plugging these estimates into (13) shows that:

$$
\sup _{\rho \in \mathcal{R}_{\kappa}^{\prime}}\left\|\widehat{\boldsymbol{\Sigma}}_{R S C M}(\rho)-\boldsymbol{\Sigma}_{N}(\rho)\right\| \stackrel{\text { a.s. }}{\longrightarrow} 0 .
$$

Finally, the handling of $\rho \in[1-\kappa, 1]$ can be carried out along the same steps as in [36. Proof of Proposition 14] by resorting to the uniform variant of the l'Hopital's rule. Details are thus omitted.

As for $\widehat{\boldsymbol{\Sigma}}_{R T E}$, the proof follows by first replacing in $\widehat{\boldsymbol{\Sigma}}_{R S C M}(\rho)$, matrix $\widehat{\mathbf{R}}_{N}^{-1}(\rho)$ by $\widehat{\mathbf{R}}_{N}^{-1}(\rho)$ and then using the convergences [35]:

$$
\sup _{\rho \in \mathcal{R}_{\kappa}^{R T E}}\left\|\frac{\widehat{\mathbf{C}}_{N}(\rho)}{\frac{1}{N} \operatorname{tr} \widehat{\mathbf{C}}_{N}(\rho)}-\widehat{\mathbf{R}}_{N}(\underline{\rho})\right\| \stackrel{\text { a.s. }}{\longrightarrow} 0,
$$

and

$$
\sup _{\rho \in \mathcal{R}_{\kappa}^{R T E}}\left|\frac{\rho}{\rho}-\frac{1}{N} \operatorname{tr} \widehat{\mathbf{C}}_{N}(\rho)\right| \stackrel{\text { a.s. }}{\longrightarrow} 0 .
$$

\section{REFERENCES}

[1] F. C. Robey, D. R. Fuhrmann, R. Nitzberg, and E. J. Kelly, "A CFAR Adaptive Matched Filter Detector," IEEE Trans. Aerosp. Electron. Syst., vol. 28, no. 1, pp. 208-216, Jan. 1992.

[2] E. Conte, M. Lops, and G. Ricci, "Adaptive Matched Filter Detection in Spherically Invariant Noise," IEEE Signal Process. Lett., vol. 3, no. 8, pp. 248-250, Aug. 1996.

[3] L. L. Scharf and L. T. McWhorter, "Adaptive matched subspace detectors and adaptive coherence estimators," Proc. IEEE Conference Record of the Asilomar Conference on Signals, Systems, and Computers, pp. 1114-1117, Nov. 1996

[4] S. Kraut, L. L. Scharf, and R. W. Butler, "The Adaptive Coherence Estimator: a Uniformly Most-Powerful-Invariant Adaptive Detection Statistic," IEEE Trans. Signal Process., vol. 53, no. 2, pp. 427-438, Feb. 2005.

[5] E. J. Kelly, "Performance of an Adaptive Detection Algorithm; Rejection of Unwanted Signals," IEEE Trans. Aerosp. Electron. Syst., vol. 25, no. 2, Mar. 1987.

[6] E. J. Kelly, "Adaptive Detection in Non-stationary Interference Part III," Technical report 761, Lincoln Laboratory, MIT, Aug. 1987, DTIC AD-A185622.

[7] F. Bandiera, D. Orlando, and G. Ricci, Advanced radar detection schemes under mismatched signal models, vol. 4, Synthesis Lectures on Signal Processing, 2009.

[8] A. De Maio, "Robust adaptive radar detection in the presence of steering vector mismatches," IEEE Trans. Aerosp. Electron. Syst., vol. 41, no. 4, Oct. 2005

[9] F. Bandiera, A. De Maio, and G. Ricci, "Adaptive CFAR Radar Detection With Conic Rejection ," IEEE Trans. Signal Process., vol. 55 , no. 6, pp. 2533-2541, June 2007.

[10] C. Hao, F. Bandiera, J. Yang, D. Orlando, S. Yan, and C. Hou, "Adaptive Detection Of Multiple Point-like Targets under conic constraints," Progress In Electromagnetics Research, vol. 129, pp. 231-250, 2012.

[11] N. B. Pulsone and C. M. Rader, "Adaptive Beamformer Orthogonal Rejection Test," IEEE Trans. Signal Process., vol. 49, no. 3, pp. 521529, Mar. 2001.

[12] E. J. Kelly and K. M. Forsythe, "Adaptive detection and parameter estimation for multidimensional signal models," Lincoln Lab, MIT, Lexington, Tech. Rep. No. 848, 1989.

[13] L. L. Scharf and B. Friedlander, "Matched Subspace Detectors," IEEE Trans. Signal Process., vol. 42, no. 8, Aug. 1994.

[14] S. Kraut, L. L. Sharf, and L. T. McWhorter, "Adaptive Subspace Detectors," IEEE Trans. Signal Process., vol. 49, no. 1, Jan. 2001.

[15] K. D. Ward, "Compound representation of high resolution sea clutter," Electronics Letters, vol. 17, no. 16, pp. 561-563, Aug. 1981.

[16] S. Watts, "Radar detection prediction in sea clutter using the compound k-distribution model," Communications, Radar and Signal Processing, IEE Proceedings F, vol. 132, no. 7, pp. 613-620, Dec. 1985.

[17] T. J. Nohara and S. Haykin, "Canadian east coast radar trials and the k-distribution," IEE Proceedings F - Radar and Signal Processing, vol. 138, no. 2, pp. 82-88, Apr. 1991. 
[18] J B Billingsley, "Ground clutter measurements for surface-sited radar," Tech. Rep. 780, MIT, February 1993.

[19] K. D. Ward, S. Watts, and R. J. A. Tough, Sea clutter: scattering, the $K$ distribution and radar performance, vol. 20, IET, 2006.

[20] P. J. Huber, "Robust estimation of a location parameter," The Annals of Mathematical Statistics, vol. 35, no. 1, pp. 73-101, 1964.

[21] P. J. Huber, "The 1972 wald lecture robust statistics: A review," Annals of Mathematical Statistics, vol. 43, no. 4, pp. 1041-1067, August 1972.

[22] R. A. Maronna, "Robust $M$-estimators of multivariate location and scatter," Annals of Statistics, vol. 4, no. 1, pp. 51-67, January 1976.

[23] E. Olilla, D. E. Tyler, V. Koivunen, and H. V. Poor, "Complex Elliptically Symmetric Distributions: Survey, New Results and Applications," IEEE Trans. Signal Process., vol. 60, no. 11, Nov. 2012.

[24] M. Mahot, F. Pascal, P. Forster, and J. P. Ovalez, "Asymptotic Properties of Robust Complex Covariance Matrix Estimates,” IEEE Trans. Signal Process., vol. 61, no. 13, pp. 3348-3356, July 2013.

[25] F. Pascal, Y. Chitour, J. P. Ovarlez, P. Forster, and P. Larzabal, "Covariance structure maximum-likelihood estimates in compound Gaussian noise: existence and algorithm analysis," IEEE Trans. Signal Process., vol. 56, no. 1, pp. 34-48, Jan. 2008.

[26] Y. Abramovich, "Controlled Method for Adaptive Optimization of Filters Using the Criterion of Maximum SNR," Radio Eng. Electron. Phys, vol. 26, no. 3, pp. 87-95, 1981.

[27] B. D. Carlson, "Covariance Matrix Estimation Errors and Diagonal Loading in Adaptive Arrays," IEEE Trans. Aerosp. Electron. Syst., vol. 24, no. 4, pp. 397-401, 1988.

[28] O. Ledoit and M. Wolf, "A Well-Conditioned Estimator for LargeDimensional Covariance Matrices," Journal of Multivariate Analysis, vol. 88, no. 2, pp. 365-411, 2004.

[29] Y. Chen, A. Wiesel, Y. C. Eldar, and A. O. Hero, "Shrinkage Algorithms for MMSE Covariance Estimation," IEEE Trans. Signal Process., vol. 58, no. 10, Oct. 2010.

[30] E. Ollila and D. E. Tyler, "Regularized M-estimators of Scatter Matrix," IEEE Trans. Signal Process., vol. 62, no. 22, Nov. 2014.

[31] Y. Chen, A. Wiesel, and O. A. Hero, "Robust Shrinkage Estimation of High Dimensional Covariance Matrices," IEEE Trans. Signal Process., vol. 59, no. 9, pp. 4907-4107, 2011.

[32] R. Couillet, F. Pascal, and J. W. Silverstein, "The random matrix regime of Maronna's M-estimator with elliptically distributed samples," Journal of Multivariate Analysis, vol. 139, pp. 56-78, 2015.

[33] R. Couillet and M. McKay, "Large Dimensional Analysis and Optimization of Robust Shrinkage Covariance Matrix Estimators," Journal of Multivariate Analysis, vol. 31, pp. 99-120, 2014.

[34] R. Couillet, F. Pascal, and J. W. Silverstein, "Robust Estimates of Covariance Matrices in the Large Dimensional Regime," IEEE Trans. Inf. Theory, vol. 60, no. 11, pp. 7269-7278, Oct. 2014.

[35] R. Couillet, A. Kammoun, and F. Pascal, "Second Order Statistics of Robust Estimators of Scatter. Application to GLRT Detection for Elliptical Signals," Journal of Multivariate Analysis, vol. 143, pp. 249274, 2016.

[36] A. Kammoun, R. Couillet, F. Pascal, and M. S. Alouini, "Optimal Design of the Adaptive Normalized Matched Filter Detector," Submitted to IEEE Transactions on Information Theory, 2015.

[37] E. Conte, A. De Maio, and G. Ricci, "Recursive estimation of the covariance matrix of a compound-Gaussian process and its application to adaptive CFAR detection," IEEE Trans. Signal Process., vol. 50, no. 8, pp. 1908-1915, Aug. 2002.

[38] F. Gini and M. Greco, "Covariance Matrix Estimation for CFAR Detection in Correlated Heavy-Tailed Clutter," IEEE Trans. Signal Process., vol. 82, no. 12, pp. 1847-1859, Dec. 2002.

[39] E. Conte, M. Lops, and G. Ricci, "Asymptotically Optimum Radar Detection in Compound-Gaussian Clutter," IEEE Trans. Aerosp. Electron. Syst., pp. 617-625, Apr. 1995.

[40] F. Pascal, Y. Chitour, and Y. Quek, "Generalized Robust Shrinkage Esitmator and Its Application to STAP Detection Problem ," IEEE Trans. Signal Process., vol. 62, no. 21, pp. 5640-5651, 2014.

[41] D. E. Tyler, "A Distribution-Free M-estimator of Multivariate Scatter," The Annals of Statistics, vol. 15, no. 1, pp. 234-251, 1987.

[42] F. Pascal and P. Forster and J. P. Ovarlez and P. Larzabal, "Performance Analysis of Covariance Matrix Estimate in Impulsive Noise," IEEE Trans. Signal Process., vol. 56, no. 6, 2008.

[43] Y. I. Abramovich and O. Besson, "Regularized Covariance Matrix Estimation in Complex Elliptically Symmetric Distributions Using the Expected Likelihood Approach: Part 1: The Over-Sampled Case," IEEE Trans. Signal Process., vol. 61, no. 23, Oct. 2013.
[44] J. W. Silverstein and Z. D. Bai, "On the empirical distribution of eigenvalues of a class of large dimensional random matrices," Journal of Multivariate Analysis, vol. 54, no. 2, pp. 175-192, 1995.

[45] Egon S Pearson, "Note on an approximation to the distribution of noncentral $\chi^{2}, "$ Biometrika, vol. 46, no. 3-4, pp. 364, 1959.

[46] A. Younsi, M. Greco, F. Gini, and A. M. Zoubir, "Performance of the Adaptive Generalized Matched Subspace Constant False Alarm Rate Detector in Non-Gaussian Noise: an Experimental Analysis," IET Radar, Sonar and Navigation, pp. 195-202, June 2009.

[47] F. Chapon, R. Couillet, W. Hachem, and X. Mestre, "The Outliers among the Singular Values of Large Rectangular Random Matrices with Additive Fixed Rank Deformation," Markov Processes and Related Fields, vol. 20, no. 2, pp. 182-228, 2014.

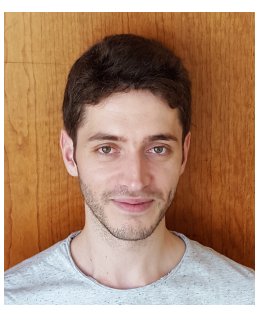

Ismail Ben Atitallah was born in Sfax, Tunisia. He received his multidisciplinary Eng. degree, Signals and Systems Minor, from Ecole Polytechnique de Tunisie, Tunisia, in 2015. He joined King Abdullah University of Science and Technology (KAUST) in August 2015, where he is currently a MS Student. His research interests include high dimensional signal processing, random matrix theory and data science.

Abla Kammoun was born in Sfax, Tunisia. She received the engineering degree in signal and systems from the Tunisia Polytechnic School, La Marsa, and the Master's degree and the Ph.D. degree in digital communications from Telecom Paris Tech [then Ecole Nationale Suprieure des Tlcommunications (ENST)]. From June 2010 to April 2012, she has been a Postdoctoral Researcher in the TSI Department, Telecom Paris Tech. Then she has been at Suplec at the Alcatel-Lucent Chair on Flexible Radio until December 2013. Currently, she is a research scientist at KAUST university. Her research interests include performance analysis, random matrix theory, and semi-blind channel estimation. She is the recepient of the SAM 2014 second Prize Best paper award.

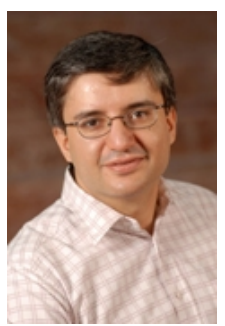

M ohamed-Slim Alouini (S'94, M'98, SM'03, F09) was born in Tunis, Tunisia. He received the Ph.D. degree in Electrical Engineering from the California Institute of Technology (Caltech), Pasadena, CA, USA, in 1998. He served as a faculty member in the University of Minnesota, Minneapolis, MN, USA, then in the Texas A\&M University at Qatar,Education City, Doha, Qatar before joining King Abdullah University of Science and Technology (KAUST), Thuwal, Makkah Province, Saudi Arabia as a Professor of Electrical Engineering in 2009. His current research interests include the modeling, design, and performance analysis of wireless communication systems.

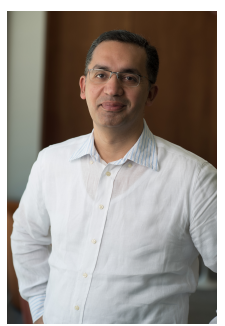

Tareq Al-Naffouri received the B.S. degrees in mathematics and electrical engineering (with first honors) from King Fahd University of Petroleum and Minerals, Dhahran, Saudi Arabia, in 1994, the M.S. degree in electrical engineering from the Georgia Institute of Technology, Atlanta, in 1998, and the $\mathrm{Ph} . \mathrm{D}$. degree in electrical engineering from Stanford University, Stanford, CA, in 2004.

He was a visiting scholar at California Institute of Technology, Pasadena, CA, from January to August 2005 and during summer 2006. He was a Fulbright scholar at the University of Southern California from February to September 2008. He has held internship positions at NEC Research Labs, Tokyo, Japan, in 1998, Adaptive Systems Lab, University of California at Los Angeles in 1999, National Semiconductor, Santa Clara, CA, in 2001 and 2002, and Beceem Communications Santa Clara, CA, in 2004. He is currently an Associate at the Electrical Engineering Department, King Abdullah University of Science and Technology (KAUST). His research interests lie in the areas of sparse, adaptive, and statistical signal processing and their applications and in network information theory. He has over 130 publications in journal and conference proceedings, 9 standard contributions, 10 issued patents, and 4 pending.

Dr. Al-Naffouri is the recipient of the IEEE Education Society Chapter Achievement Award in 2008 and Al-Marai Award for innovative research in communication in 2009. Dr. Al-Naffouri has also been serving as an Associate Editor of Transactions on Signal Processing since August 2013. 